\title{
The search campaign to identify and image the Philae Lander on the surface of comet $67 \mathrm{P} /$ Churyumov-Gerasimenko ${ }^{\text {ir }}$
}

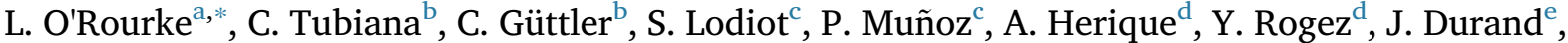 \\ A. Charpentier ${ }^{\mathrm{f}}$, H. Sierks ${ }^{\mathrm{b}}$, P. Gutierrez-Marques ${ }^{\mathrm{b}}$, J. Deller ${ }^{\mathrm{b}}$, B. Grieger ${ }^{\mathrm{g}}$, R. Andres ${ }^{\mathrm{h}}$, B. Geiger ${ }^{\mathrm{g}}$, \\ K. Geurts ${ }^{\mathrm{i}}$, S. Ulamec ${ }^{\mathrm{i}}$, N. Kömle ${ }^{\mathrm{j}}$, V. Lommatsch ${ }^{\mathrm{i}}$, M. Maibaum ${ }^{\mathrm{i}}$, J.L. Pellon ${ }^{\mathrm{k}}$, C. Bielsa ${ }^{1}$, \\ R. Garmier ${ }^{\mathrm{m}}$, M. Taylor ${ }^{\mathrm{n}}$, P. Martin ${ }^{\mathrm{a}}$, M. Küppers ${ }^{\mathrm{a}}$, A. Accomazzo ${ }^{\mathrm{c}}$, V. Companys ${ }^{\mathrm{c}}$, J.P. Bibring ${ }^{\circ}$, \\ W. Kofman ${ }^{\text {, }}$ S. Mckenna Lawlor ${ }^{\mathrm{p}}$, M. Salatti ${ }^{\mathrm{q}}$, P. Gaudon ${ }^{\mathrm{e}}$
}

${ }^{a}$ European Space Astronomy Centre, European Space Agency, Madrid, Spain

${ }^{\mathrm{b}}$ Max Planck Institute for Solar System Research, Göttingen, Germany

${ }^{\mathrm{c}}$ European Space operations Centre, European Space Agency, Darmstadt, Germany

${ }^{\mathrm{d}}$ Institut de Planétologie et d'Astrophysique de Grenoble, Grenoble, France

e Centre National d'Etudes Spatiales (CNES), France

${ }^{\mathrm{f}}$ ATOS, Toulouse, France

${ }^{g}$ Aurora Technology B.V., ESAC, Madrid, Spain

${ }^{\mathrm{h}}$ Telespazio Vega UK Limited, ESAC, Madrid, Spain

${ }^{\mathrm{i}}$ Deutsches Zentrum für Luft, und Raumfahrt e.V. (DLR), Germany

j ÖAW, Space Research Institute, Austrian Academy of Sciences, Vienna, Austria

${ }^{\mathrm{k}}$ European Space operations Centre, Darmstadt, Germany

${ }^{1}$ SCISYS Deutschland Gmbh, Darmstadt, Germany

${ }^{\mathrm{m}}$ CS-SI Communication \& Systèmes, Toulouse, France

${ }^{\mathrm{n}}$ European Space Research \& Technology Centre, European Space Agency, Noordwijk, the Netherlands

${ }^{\circ}$ IAS, Orsay, France

${ }^{\mathrm{p}}$ Space Technology Ireland Limited, Maynooth, Ireland

${ }^{\mathrm{q}}$ Italian Space Agency, Italy

\section{A B S T R A C T}

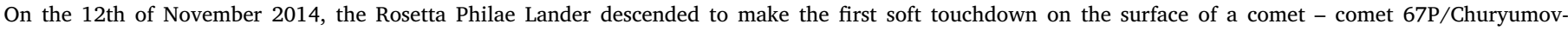

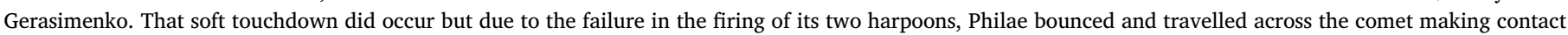

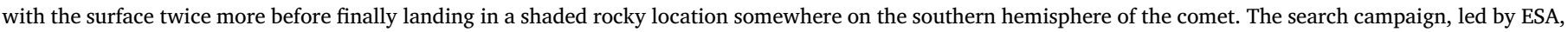

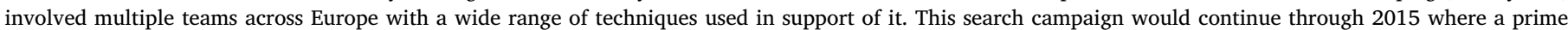

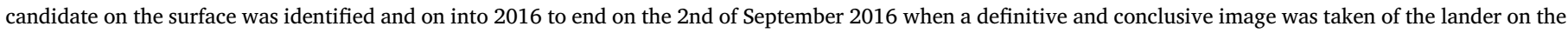
surface of the comet, confirming the prime candidate to indeed be Philae.

\section{The Philae landing - a bounce into the unknown}

The Rosetta mission represented a Cornerstone of the ESA Horizon 2000 programme [1]. It was launched in March 2004 and arrived at its prime destination comet 67P/Churyumov-Gerasimenko (CG) in August 2014, after a 10 year cruise across the solar system. After arrival, activities immediately kicked off to select a landing site for the Philae Lander that had travelled the long journey with Rosetta [2]. With the landing site "Agilkia" chosen in September 2014 (Fig. 1), steps then took place to prepare for the separation of Philae a number of weeks later.

Philae separated from Rosetta in the early hours of 12th of November 2014 and drifted down to the surface during a seven-hour descent phase. Communication between Rosetta and Philae took place using the "Electrical Support System" (ESS) located on Rosetta [3]. Contact with the ESS was established between Rosetta and Philae about

\footnotetext{
Rosetta is an ESA mission with contributions from its member states and NASA. Rosetta's Philae Lander is provided by a consortium led by DLR, MPS, CNES and ASI with additional contributions from Hungary, UK, Finland, Ireland and Austria.

* Corresponding author.

E-mail address: lorourke@esa.int (L. O'Rourke).
} 


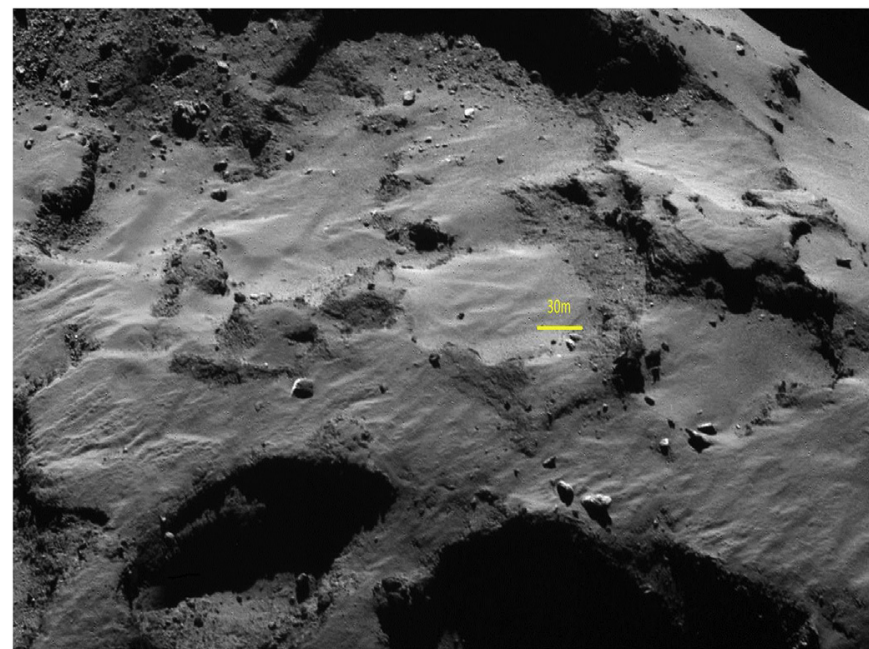

Fig. 1. Agilkia landing site - Centre of the image - image taken on the $30^{\text {th }}$ September 2014 at a distance of $16 \mathrm{~km}$ from the landing site. Credits: ESA/ Rosetta/MPS for OSIRIS Team MPS/UPD/LAM/IAA/SSO/INTA/UPM/DASP/ IDA.

$1 \mathrm{~h}$ after separation to then allow the touchdown to be monitored by the control centres located at ESOC (European Space Operations Centre) for the Rosetta Spacecraft and at DLR (Deutsches Zentrum für Luft-und Raumfahrt) for the Philae Lander.

At touchdown, the lander's harpoons failed to fire leading to Philae bouncing and rising once again from the surface. It travelled across the surface for a period of $2 \mathrm{~h}$, touching the surface twice more before finally setting down in a region over one kilometre away from Agilkia [4].

Philae was operational throughout the full descent and bounce phases and was to continue for another $63.73 \mathrm{~h}$ providing unique in-situ science from its ten instruments [5]. Images taken by the CIVA camera [6] provided valuable information as to the local terrain surrounding Philae.

The communication [7] between lander and orbiter via the ESS gave clues as to the direction of the lander with respect to the Rosetta orbiter. Further to this, data from the CONSERT (Comet Nucleus Sounding Experiment by Radiowave Transmission) instrument, installed on both Philae and on Rosetta, served as a key input to help locate Philae's position in a region on the edge of the Hatmehit depression. At this stage, with Philae entering into hibernation, its exact location on the surface was however yet to be determined.

This paper gives a detailed description of the steps taken to locate and image Philae on the surface of the comet. Section II provides a list of the different centres involved in the various lander search campaigns that took place through late 2014 to early September 2016. Section III introduces the different Rosetta and Philae instruments/subsystems used during the lander search. Section IV presents the work carried out to find Philae from the time of the landing on 12th of November 2014 up to the time when Philae went into hibernation on the 15th of November 2014. Section V presents the steps performed during the second search campaign leading up to June 2015. Section VI presents the third search campaign, which included the wake-up of Philae, covering the period from June 2015 up until March 2016. Section VII then closes out the search for Philae by describing the fourth and final search campaign that led to the famous Philae image taken just a few weeks before the Rosetta end of mission. We end the paper with a short wrap-up and conclusions section.

\section{The different centres involved in the lander search}

A number of centres and groups across Europe took part in the search for and imaging of Philae on the surface of the comet. Depending on the search campaign in question, their role and activities differed.

In many cases e.g. CONSERT, although individuals from a number of collaborative institutes were also involved only the prime institute is listed below; these other institutes are listed in the acknowledgements section of this paper. Further to this, the number of co-authors of this paper represent only a select set of those who participated in the Lander search - all names are provided in the acknowledgements section.

The list below defines therefore the prime interface that existed with ESA during the search campaign.

- CONSERT - Comet Nucleus Sounding Experiment by Radiowave Transmission - Principal Investigator located in IPAG Université Grenoble Alpes, Grenoble, France

- IRAP - Institut de Recherche en Astrophysique et Planétologie, France

- LAM - Laboratoire d'Astrophysique de Marseille, France

- LCC - Philae Lander Control Centre team at DLR, Cologne, Germany

- OSIRIS - Optical, Spectroscopic, and Infrared Remote Imaging System team in MPS, Göttingen, Germany

- PSC - Philae Lander Steering Committee

- RMOC - ESA's Rosetta Mission Control Centre in ESOC, Darmstadt, Germany

- ROMAP-MAG - Rosetta Lander Magnetometer and Plasma Monitor, Technische Universität, Braunschweig, Germany

- RSGS - ESA's Rosetta Science Ground Segment located in ESAC, Madrid, Spain

- SONC - Rosetta Science Operations and Navigation Centre at CNES

SWT - Rosetta Science Working Team (SWT) led by the ESA project scientist

\section{The spacecraft systems used in support of the search campaigns}

Throughout this paper reference is made to different Rosetta and Philae instruments and subsystems that supported the search campaigns. For the sake of completeness we introduce them below with more detailed information provided in later sections outlining how they were used in the different search campaigns.

The following instruments and subsystems were present on the Rosetta Orbiter:

- The OSIRIS instrument [8] comprised two cameras: a high resolution Narrow Angle Camera (NAC) and a Wide Angle Camera (WAC). Both are unobstructed off-axis mirror systems, equipped with two filter wheels containing 8 positions each, and with backside illuminated CCD detectors comprising $2048 \times 2048$ pixels with a pixel size of $13.5 \mu \mathrm{m}$.

- The NAVCAM (Navigation Camera) [9] comprised two cameras each with $5^{\circ}$ field of view and 12 bit $1024 \times 1024 \mathrm{px}$ resolution which allowed for visual tracking as the spacecraft approached and orbited the comet

- The ESS (Electrical Support Subsystem) [3] was mounted on the Rosetta spacecraft. It wrapped telecommands from Rosetta in a transfer frame for forwarding via the RF link to the Philae Lander. Telemetry data from the Lander experiments uploaded via the RF link to Rosetta, was received and formatted by the ESS.

The following instruments were located on the Philae Lander

CIVA (the Comet Infrared and Visible Analyser) [10] was a set of seven identical cameras that produced a panoramic image from Philae's resting site. CIVA was designed to characterise the landing site, mapping the surface topography and the albedo (reflectivity) of the surface. Two of the cameras were aligned in order to produce stereoscopic images.

ROMAP [11] was a combination of a magnetometer and plasma 
instrument with high temporal and spatial resolution.

The following instruments and subsystems were located on both the Rosetta Orbiter and the Philae Lander.

- The CONSERT radar [12] performed tomography of the nucleus by measuring electromagnetic wave propagation from the Philae Lander and the Rosetta Orbiter throughout the comet nucleus in order to determine its internal structures and to deduce information on its composition

- Radiofrequency links [1] - there were two transceivers on both sides (Rosetta and Philae) of the RF link. Each transceiver was composed of a transmitter, a receiver and a reception filter for dual antenna use. Use of the different RF chains was possible by activating RF switches on the orbiter side ( $1 \mathrm{Tx} / 1 \mathrm{Rx}$ active) and the diplexer on the Philae lander side ( $1 \mathrm{Tx} / 2 \mathrm{Rx}$ active)

\section{The first search campaign - what we knew 3 days after landing}

The Philae Lander touched the surface of comet CG at 15:34 UTC on the 12th of November 2014. Upon touchdown, Philae was expected to fire two harpoons into the surface to aid in anchoring the lander. A thruster on the top of the lander was also expected to fire in order to compensate the momentum caused by harpoon firing and to push the lander towards the surface at the same time. Both the harpoons and the thruster did not fire however leading to the Philae lander bouncing off the surface and travelling uncontrolled for almost $2 \mathrm{~h}$ more before it finally stopped having lodged in a dark "rocky" region located over one kilometre away.

With Philae alive and communicating with the Rosetta orbiter throughout its bounce as well as after it had reached its final location [4], an immediate effort was made by the teams involved to identify where Philae was located before the lander's battery was depleted and it entered hibernation. Hibernation took place on the 15th of November 2014.

This first search campaign (see Table 1) used inputs from the actual data of the lander (CIVA cameras, Philae Radio Frequency (RF) signals, Philae Solar Arrays, ROMAP), as well as data from the CONSERT instrument.

The CIVA camera images [6] helped to give ground truth information as to the final location of Philae. It became clear from the images obtained that Philae had landed in a shadowed rocky location below a cliff overhang.

Eleven hours after the initial touchdown the CONSERT team published a first orbital stripe crossing the surface of the comet. Eight hours later this stripe was fine-tuned leading to Fig. 2 below. This first localisation was based on radar signals propagated throughout the comet nucleus.

In the days following the landing, the Rosetta project allocated additional observational time to CONSERT during RF visibility to improve localisation. At the same time, the reconstruction of Rosetta orbital data "moved" the triangulated position to the south, outside the Hatmehit depression (Fig. 3). By the 15th November 2014, further orbital stripes due to additional measurements between CONSERT on the lander and CONSERT on the orbiter were added (Fig. 3) leading to a high level "X marks the spot" view of the landing location. By the time
Philae went into hibernation, its general location on the surface of the comet had been identified with an uncertainty of only about $100 \times 30 \mathrm{~m}^{2}$.

\section{The second search campaign - results obtained up to June 2015}

The analysis of both CONSERT data in visibility and in transmission were refined in the months after hibernation in order to improve the accuracy and estimate uncertainties. Results were furnished to operation centres to support lander search campaigns [13,21,22].

A couple of weeks after hibernation, a first reconstruction of Philae's trajectory after touch-down was provided by ESOC Flight Dynamics [14].

During these weeks after hibernation, significant effort was spent in studying the images to try to identify Philae on the surface. In reality it was quickly seen that there were three main challenges making rapid identification difficult.

The first was the reflectivity of the solar panels of Philae which was comparable to that of ice on the surface of the comet, thus making it difficult to differentiate between ice and the lander in the images. The second related to the actual size of Philae in the available images. With the body of Philae measuring about $1 \mathrm{~m}$ across and its three legs extending out by up to $1.5 \mathrm{~m}$ from its centre, images taken after landing at a distance of $20-25 \mathrm{~km}$ would lead to Philae measuring only 2-3 pixels across in the OSIRIS Narrow Angle Camera (NAC) field of view. The third challenge related to the orientation and location of Philae on the surface whereby it was positioned on its side in an ice rocky region meaning that only part of the lander would be illuminated.

By the 25th of November 2014, a further fine tuning of the location of Philae was published by the CONSERT team (Fig. 4) leading to an area just off the edge of the Hatmehit depression. With this location in mind, a lander imaging campaign took place in mid-December 2014 when Rosetta approached sufficiently close (approx. $18 \mathrm{~km}$ from the surface) to allow higher resolution images to be taken. At this distance, the OSIRIS NAC had a resolution of $30 \mathrm{~cm} /$ pixel meaning that the image of Philae would be a few pixels across.

Following reception of these new images and the identification of possible Philae candidates, scientists at the Laboratoire d'Astrophysique de Marseille (LAM) and the Institut de Recherche en Astrophysique et Planétologie (IRAP) in France began searching through all OSIRIS images existing up to that point of the region in question. In particular, they looked for images taken before and after Philae's landing that had similar viewing geometry and illumination conditions as those just received; the goal was to reduce the chances of being misled by transient surface features glimmering in the sunshine. In that way, in principle, if something new appeared after landing, it had a high chance to be Philae.

Scanning a broad area encompassing the expected landing zone, five candidates were identified (see Fig. 5). The candidate on the top left of Fig. 5 was chosen by the LAM team to be the most promising candidate from the five. This candidate (titled the LAM candidate) was seen on two images taken during the first search campaign on the 12th and 13th of December 2014 but not in an image taken before landing on the 22nd of October 2014 [15].

A closer study of the before and after images (see Fig. 6) found however that the position of the Philae lander in the October image was

Table 1

Search Campaign overview.

\begin{tabular}{|c|c|c|c|}
\hline Search Campaign Number & Campaign Start Date & Campaign End Date & Figures in the paper per campaign \\
\hline 1 & 12th of November 2014 & 15th of November 2014 & Figs. 2 and 3 \\
\hline 2 & 16th of November 2014 & 12th of June 2015 & Figs. 4-6 \\
\hline 3 & 13th of June 2015 & 31st of July 2015 & Fig. 7 \\
\hline 4 & 9th of March 2015 & 2nd of September 2016 & Figs. 8-24 \\
\hline
\end{tabular}




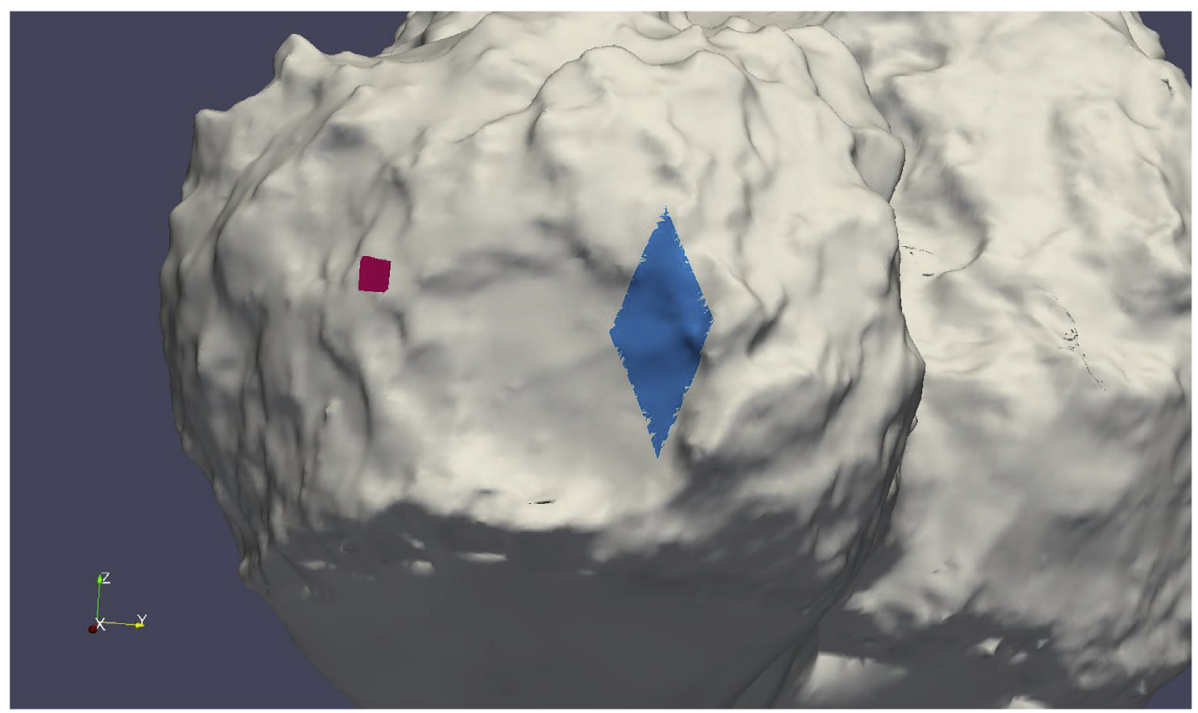

Fig. 2. Search Campaign 1 - Blue diamond identifies the lander location according to the CONSERT estimation provided $17 \mathrm{~h}$ after touchdown in the Agilkia location (shown in red). Credits: Diamond: ESA/ Rosetta/Philae/CONSERT; Shape model: ESA/ Rosetta/MPS for OSIRIS Team MPS/UPD/LAM/IAA/ SSO/INTA/UPM/DASP/IDA. (For interpretation of the references to colour in this figure legend, the reader is referred to the Web version of this article.)

shadowed. In addition, the fact remained that as seven weeks had passed since the October image and the December image, one could not discard the likelihood that this was recently exposed ice on the comet surface. Because of this a definitive statement that this was indeed unequivocally the Philae lander could not be given.

In parallel to the above work, the SONC team at CNES, had identified another possible landing location only $14 \mathrm{~m}$ away from the defined LAM candidate position. Their analysis [16] had taken into account data such as the CONSERT most probable area of landing, the attitude as proposed by ROMAP, the housekeeping data for illumination of the solar array and RF communications. This difference of $14 \mathrm{~m}$ can be explained however by the differing shape models (and associated resolution of position) in use during the analysis by both teams.

Of importance to note at this point is that a reconstruction of the Digital Terrain Model (DTM) where they placed a 3D model of Philae in the LAM location allowed the LAM team to make a good comparison with the actual CIVA images taken on the comet surface $[17,18]$.

With the small error bar between the LAM \& CNES candidate positions and the DTM analysis, it was decided for later search campaigns to consider only the LAM Candidate position to be the prime search location.

\section{Philae re-awakens and the third search campaign begins}

Using as input the power \& thermal measurements taken before Philae entered hibernation in November 2014, with models based on its likely location and the comet's orbit, it was decided to commence a Philae wake-up campaign in March 2015 during selected periods where Rosetta's orbital geometry was favourable to communication. The spacecraft distance to the comet ranged from 90 to $70 \mathrm{~km}$ during this period.

The power and thermal estimates produced by the Philae team concluded that by the March/April 2015 timeframe, Philae would have an internal temperature above $-45{ }^{\circ} \mathrm{C}$ and its battery charged sufficiently to provide the more than $5.5 \mathrm{~W}$ of power needed to be able to at least boot up. With that said, RF communication could only be possible once the internal power had reached $19 \mathrm{~W}$.

The goal of this wake-up campaign was to carry out the "long term science" phase on the Philae Lander based upon use of the secondary batteries charged from the solar panels.

In order to establish communication with Philae, the ESS on the Orbiter, responsible for the interface between the Rosetta and Philae, was switched on in early March 2015 and configured in "Research Mode". In this mode, the ESS transmitter sent signals to Philae while the

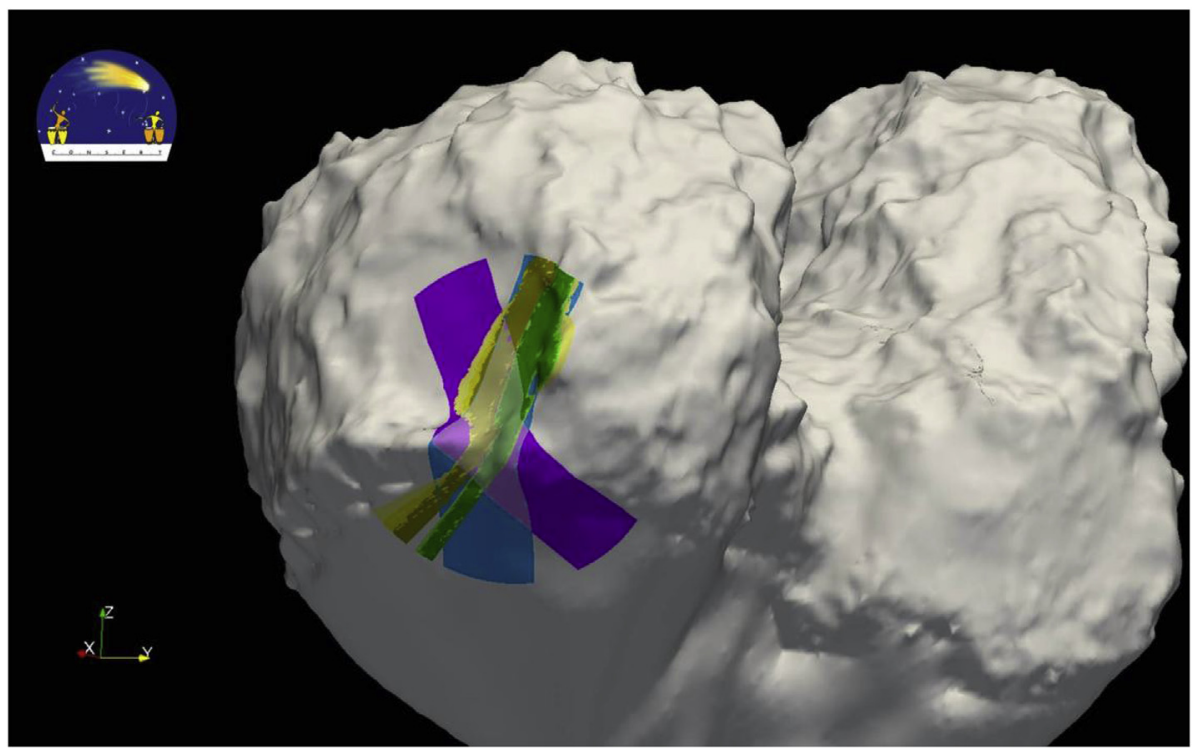

Fig. 3. Search Campaign 1 - Further Consert estimations after landing showing the overlaps of the orbital data on the comet surface. The smooth region on the shape model corresponds to the southern part of the comet which was not yet mapped at time of shape model production. Credits: Stripes: ESA/ Rosetta/Philae/CONSERT; Shape model: ESA/ Rosetta/MPS for OSIRIS Team MPS/UPD/LAM/IAA/ SSO/INTA/UPM/DASP/IDA. 


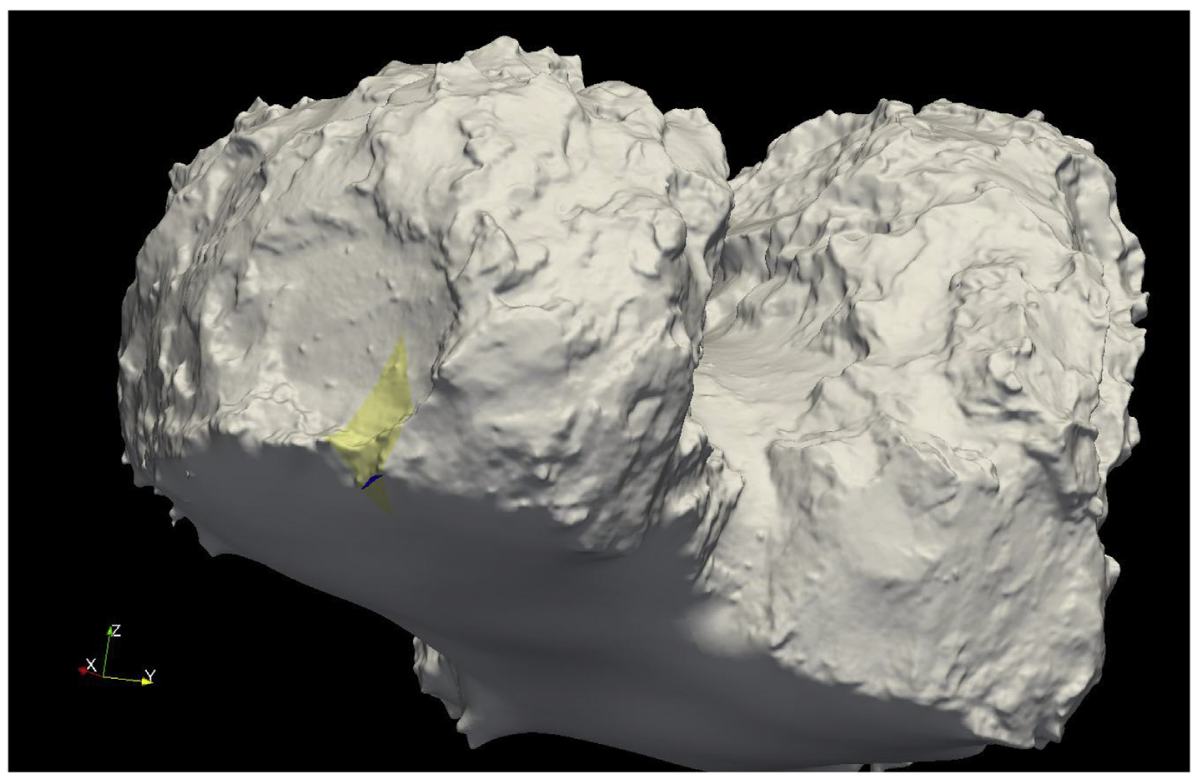

Fig. 4. Search Campaign 2 - Consert localisation as estimated on 25th November 2014. Credits: Yellow overlays: ESA/Rosetta/Philae/CONSERT; Shape model: ESA/Rosetta/MPS for OSIRIS Team MPS/ UPD/LAM/IAA/SSO/INTA/UPM/DASP/IDA. (For interpretation of the references to colour in this figure legend, the reader is referred to the Web version of this article.)

ESS receiver listened for a reply [19].

In such a scenario, if the ESS signal was received by either of the receivers on Philae, the on-board software would perform a check to see if there was enough power (19 W) [20] to switch on one of its two redundant transmitters. If so, the transmitter turns on and sends a signal, with a specific pattern, to the ESS on Rosetta telling it that it is ready to establish a two-way link. Upon establishment of the resulting two-way link, Philae could immediately start uploading science and housekeeping data that was stored in its on-board mass memory (MM). Rosetta could also send new command sequences for Philae to load and execute, initiating scientific measurements, for example.

Unfortunately, although the ESS was attempting to communicate with Philae, the spacecraft distance had to increase well beyond $200 \mathrm{~km}$ in late March due to safety constraints. This greater distance significantly hampered the communication capabilities that were possible with the Philae Lander. With no signal received for the following number of months, it was decided on the 12th of June 2015 to carry out a dedicated search campaign for Philae whereby Rosetta's trajectory would be optimised for possible contacts with Philae. This campaign was due to start mid-July but in reality fate took over as the Philae signal was received on the 13th June [19].

On the 13th of June 2015, with Rosetta flying at a distance of $200 \mathrm{~km}$ from the surface, it passed over the region where Philae was located and established communication with the lander. The third search campaign had thus begun (see Table 1). At signal reception, ESA's Rosetta Mission Operations Centre (RMOC) changed the trajectory flown by Rosetta while attempting to get closer to the comet in order to maximise the possibilities of communication. Communication attempts were feasible twice daily.

Mindful of the concerns regarding the star trackers and the dusty environment that led to the late March safety concerns, the RMOC team nevertheless pushed Rosetta towards its safe operational limits, stepping down the distance to the comet every 3-4 days in late June and early July. For example, the contact made on the 24th of June was at a distance of $180 \mathrm{~km}$, while the next contact was at $155 \mathrm{~km}$ on 9th of July; see also [19, table 3 for the full list of contacts]. During the initial Philae on-comet operations in November 2014, Rosetta had been below $30 \mathrm{~km}$ however the majority of the time.

In addition to the reducing distance, the trajectory of Rosetta and the rotation of CG meant that Rosetta appeared over the region where

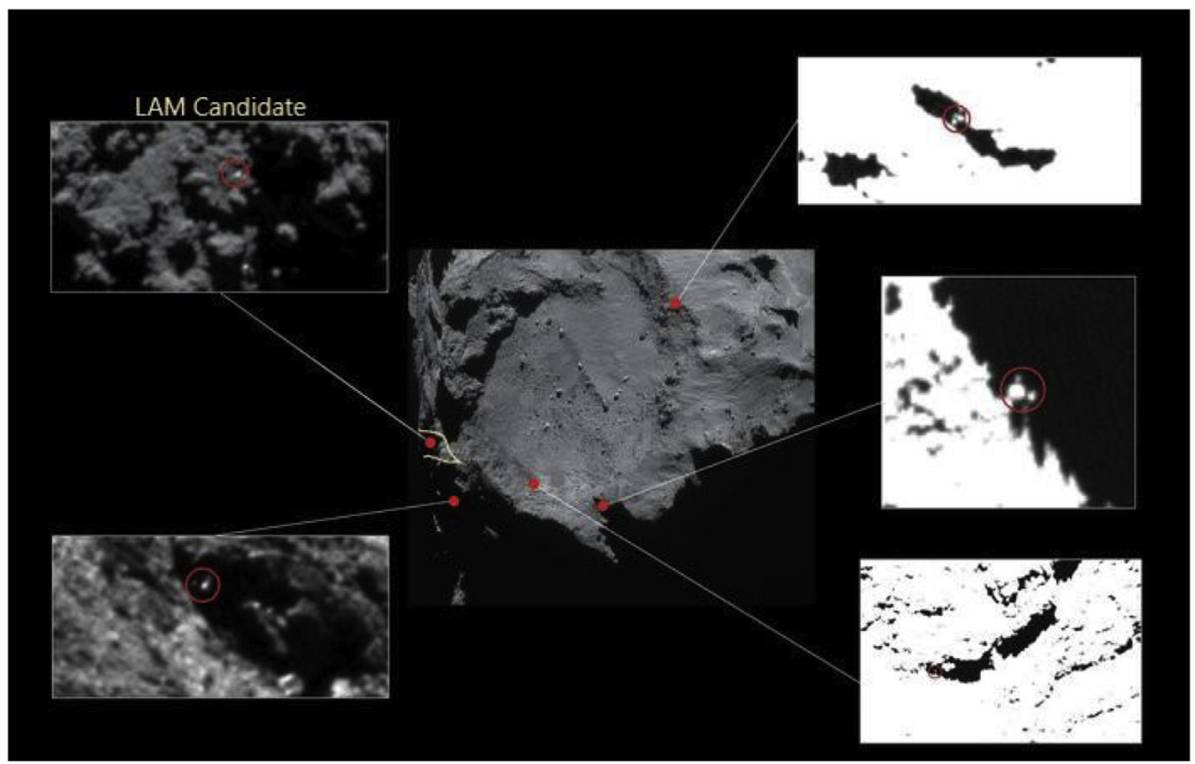

Fig. 5. Search Campaign 2 - Approximate locations of five lander candidates initially identified in highresolution images taken in December 2014, from a distance of about 12.4 miles $(20 \mathrm{~km})$ from the comet's center. The candidates identify Philae-sized features about 3-6 feet (1-2 m) across. The contrast has been stretched in some of the images to better reveal the candidates. All but one of these candidates (top left) were subsequently ruled out. The candidate at top left lies near to the current CONSERT ellipse (see Fig. 4 and 6) the edge of which is marked in yellow; this candidate was the LAM candidate which is shown further in Fig. 6. (For interpretation of the references to colour in this figure legend, the reader is referred to the Web version of this article.) NOTE: This figure provides an overhead view of the Hatmehit depression whereby most candidates can be found located on its southern edge. It is important to flag that the lighting conditions and orientation of this image differs to that of the shape model shown in Fig. 4. Credit: ESA/Rosetta/MPS for OSIRIS Team MPS/UPD/LAM/IAA/SSO/INTA/UPM/DASP/IDA. 

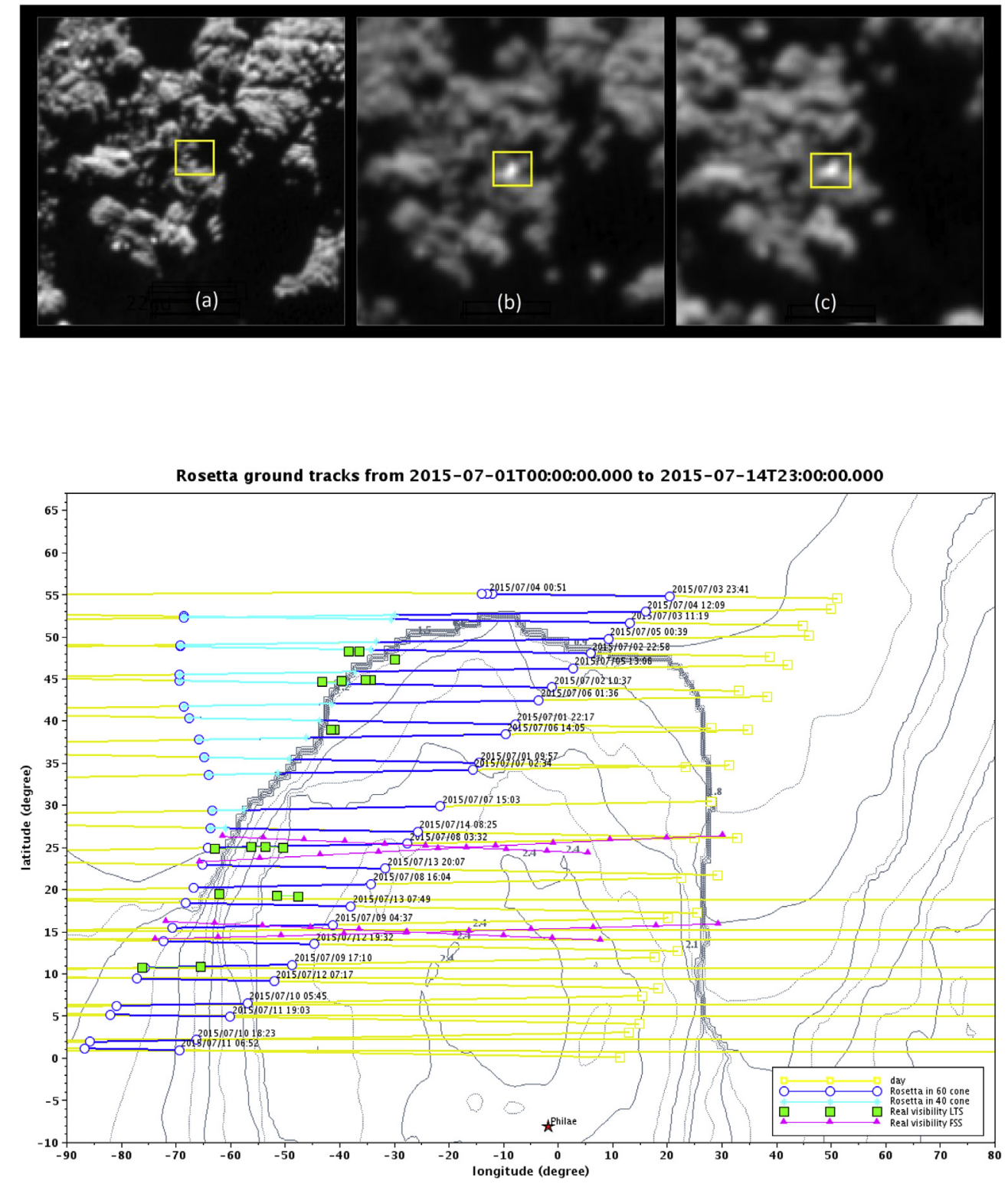

Fig. 6. Search Campaign 2 - images show the region containing the candidate as seen (a) on 22nd of October 2014 at a distance of $8 \mathrm{~km}$ from the surface (Philae would be over 5 pixels in size), and then on both (b) 12th of December 2014 and (c) 13th of December 2014 at a distance of $18 \mathrm{~km}$ from the surface (Philae would be 3 pixels in size). The location of Philae is identified within the yellow square; in the left image one can observe the location is primarily in shadow. Credits: ESA/Rosetta/MPS for OSIRIS Team MPS/UPD/LAM/IAA/SSO/INTA/UPM/

DASP/IDA. (For interpretation of the references to colour in this figure legend, the reader is referred to the Web version of this article.)

Fig. 7. Search Campaign 3 - Computation of Rosetta's trajectory with respect to the comet surface (yellow and blue lines) and the established contacts with Philae in November (pink lines) and June/July (green squares). The map shows a portion of the comet surface on the small lobe; the large circular feature visible in the central lower part of the map is in the Hatmehit region - The depression Hatmehit is roughly circular, with a diameter about a kilometer and a depth of $150-300 \mathrm{~m}$. Philae is assumed to be located on bottom centre. Credits: CNES/SONC Flight Dynamics. (For interpretation of the references to colour in this figure legend, the reader is referred to the Web version of this article.)
Philae was believed to be located, at different comet latitudes each comet day. Over these few weeks, the range in latitudes between $0^{\circ}$ and $+55^{\circ} \mathrm{N}$ was covered several times, see Fig. 7 .

As communication between Rosetta and Philae did not take place every comet day (every $12 \mathrm{~h}$ ), as was expected, it was hypothesised that there was a dependency on latitude. However, even so, contacts did not take place at the same latitudes each time, and local topographical features were believed to also obstruct the signal in some cases, thus complicating the analysis. In addition, it was not clear why the contacts - when established - were so unstable. Overall, it was proving hard for the team to come up with reliable predictions as to when good contacts would occur.

On the 9th of July 2015, a final connection was successfully established with Philae and a study of the data received concluded that the lander had problems with its transmitters. This final connection lasted $22 \mathrm{~min}$ (the longest duration of all contacts) of which $12 \mathrm{~min}$ were a solid signal. In hindsight, it is worth pointing out that later analysis of the horizon mask (RF visibility) would show that the break in the link at the end of the 22 min was because Rosetta had flown behind the ridge of the Hatmehit depression. During this 9th of July 2015 communication session, attempts to switch on and establish contact with the CONSERT antenna proved to be unsuccessful.

Once this communication ended, no further communication was to occur between Philae and Rosetta for the remainder of the mission. The communication established during this period did serve however to confirm that Philae had not moved from its original landing location. Good news leading up to the perihelion passage where the comet would undergo its maximum activity level.

\section{The fourth and final search campaign}

\subsection{The prime "red" and "alternate" blue candidates}

By July 2015, the CONSERT team published their radar data [21] triangulating the location to the general area now known as Abydos with a further more accurate calculation published in November 2015 [22]. The region of the lander location as predicted by CONSERT is shown in Fig. 8 below.

In August 2015, Rosetta and the comet reached perihelion. With the star trackers of Rosetta becoming at times overwhelmed by the quantity of dust produced (shining like stars in its field of view), Rosetta had to move further and further from the comet thus preventing any feasible 


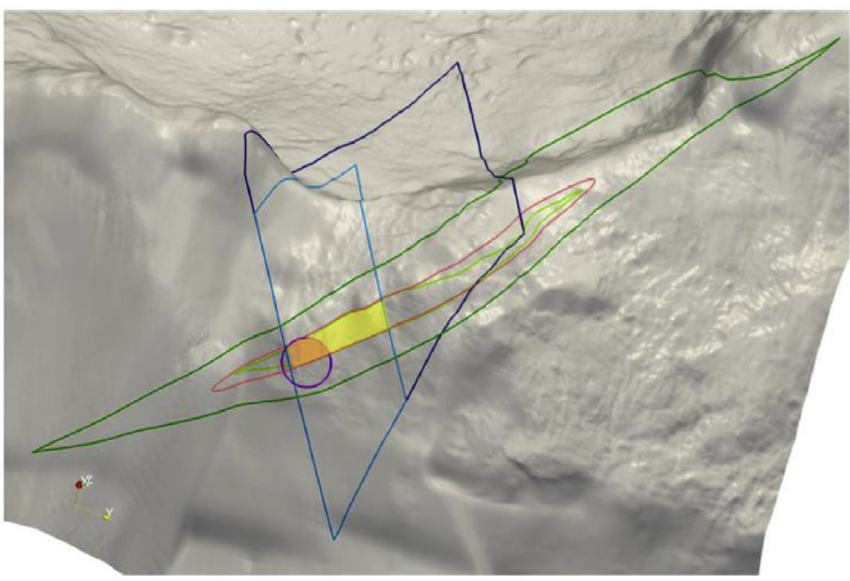

Fig. 8. Search Campaign 4 - Lander localisation from CONSERT data [22]: Projected location of Philae on the surface of $67 \mathrm{P}$. Light green polygon: line of sight measurements $\min / \max \pm 1$ sigma. Dark green polygon: $\mathrm{min} / \mathrm{max}$ distance \pm 3 sigma. Light blue: propagation through the comet at a maximum of $325 \mathrm{~m}$ and \pm 3 sigma. Dark blue: propagation through the comet at a maximum of $1000 \mathrm{~m}$ and \pm 3 sigma. Red ellipse: zone with RMS error below 1.0. Purple circle: from joint inversion of permittivity and position. Yellow zone: taking into account all measurements $\left(22.5 \times 106.5 \mathrm{~m}^{2}\right)$ Orange zone. The most likely estimate $\left(22.5 \times 41.5 \mathrm{~m}^{2}\right)$. Credits: ELLIPSE: ESA/ROSETTA/PHILAE/CONSERT; SHAPE MODEL: ESA/ROSETTA/MPS FOR OSIRIS TEAM MPS/UPD/ LAM/IAA/SSO/INTA/UPM/DASP/IDA. (For interpretation of the references to colour in this figure legend, the reader is referred to the Web version of this article.)

communication to take place with Philae. It was thus only in March 2016 that the comet's activity finally granted Rosetta's safe passage to within $30 \mathrm{~km}$ of the nucleus again. At this point, the fourth and last search campaign kicked off (see Table 1).

On the 9th of March 2015, an image taken (Fig. 9) served as the key for the fourth search campaign kick-off in that two candidates were identified for follow-up. The first was the prime LAM candidate (referred in the coming sections as the "red" candidate to match with the colour scheme in Fig. 9). The second was an alternate candidate and referred to as the "blue" candidate (also shown in Fig. 9).

As described in an earlier section, the LAM or "red candidate" lay within the CONSERT ellipse and appeared to meet the various power,
$\mathrm{RF}$ and visibility constraints linked to the actual data from the lander, along with showing promising correlation between CIVA and OSIRIS images.

The alternate "blue candidate" however was located on the southern face of the comet at a distance of approximately $400 \mathrm{~m}$ from the Consert ellipse (where the red candidate lies). While a zoom in on this candidate does show a good match with the characteristic shape of Philae (Fig. 9 inset), for this to be Philae it would need to have moved from the Consert ellipse to this other location. Based upon the fact that the comet had now passed through the perihelion passage, such a move could not be ruled out.

The fourth campaign kicked off therefore with the goal to obtain high resolution images of the red and blue Philae candidates from different distances and viewing angles around the comet. This addressed two issues: the first being that the lander could be blocked by a rock from one direction while visible from another direction, and the second that such an approach allowed different illumination conditions and phase angles to be taken into account.

\subsection{The devil is in the detail: identifying and planning images}

Once the trajectory to be flown by Rosetta was agreed, it was analysed by various teams involved in the lander search to identify the possible times where there would be line-of-sight visibility between Rosetta and the Philae candidates, as well as ensuring it would be wellilluminated (see Fig. 10).

Fig. 11 provides an example of how the windows were provided for the detailed analysis/selection to take place.

As the science phase of the mission was in full swing, care was taken to choose opportunities based upon factors such as:

- Minimising impact on key orbiter science observations planned during those periods

- Avoiding times where the orbiter/Sun was below $20^{\circ}$ from the horizon

- Avoiding times where spacecraft manoeuvres took place

- Avoiding times where the spacecraft pointing error was predicted to be high

- Minimising repetition where the field of view was similar at different epochs

- Using spacecraft maintenance window periods where feasible

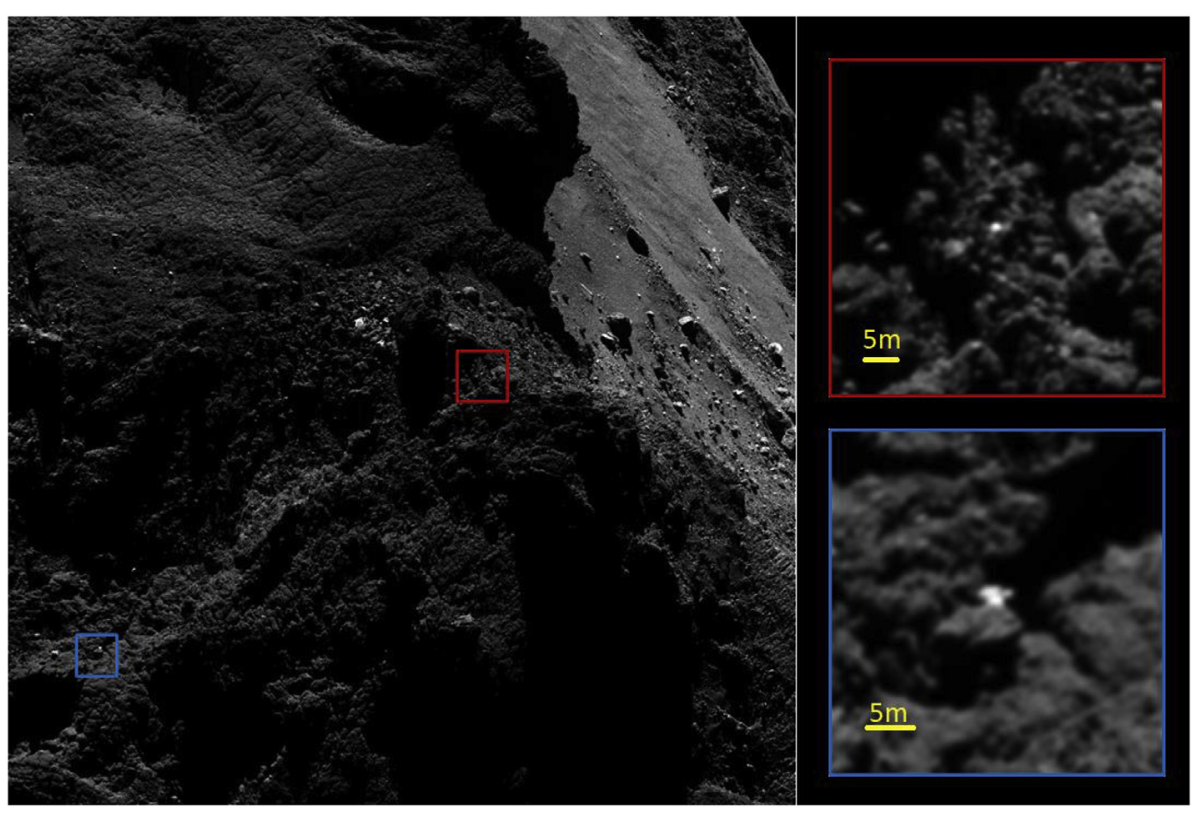

Fig. 9. Search Campaign 4 - The prime (Red) and (alternate (blue) marked candidates in an OSIRIS NAC image taken on the 9th of March 2016 at a distance of $13.8 \mathrm{~km}$. Credits: ESA/Rosetta/MPS for OSIRIS Team MPS/UPD/LAM/IAA/SSO/INTA/ UPM/DASP/IDA. (For interpretation of the references to colour in this figure legend, the reader is referred to the Web version of this article.) 


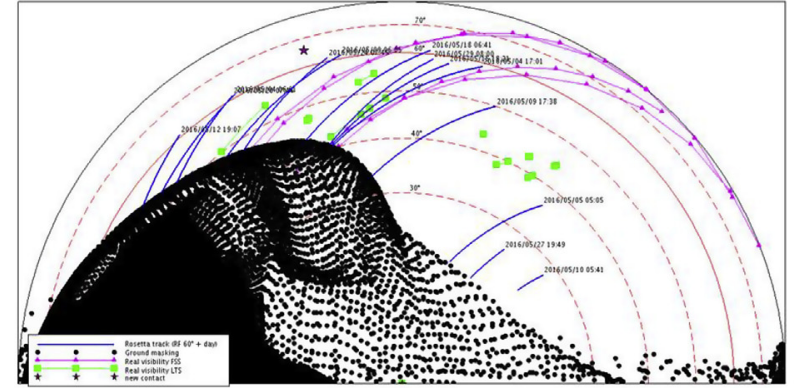

SONC (CNES) Orbiter Visibility Windows (May 2016)

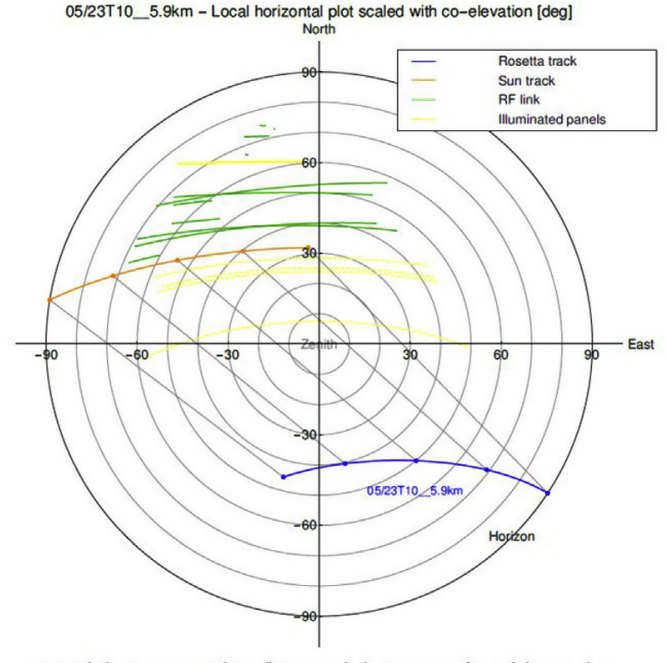

ESOC Flight Dynamics Orbiter \& Sun track during a specific visibility window (Red Candidate at centre of plot)

Fig. 10. Search Campaign 4 - Two different ways of viewing the orbiter flight trajectory when compared to the lander position.

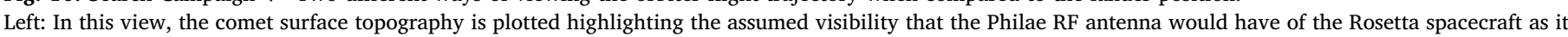

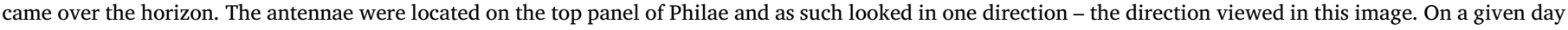

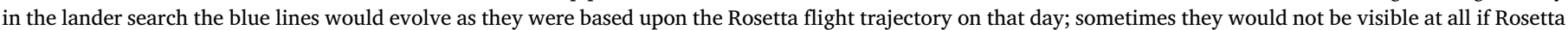

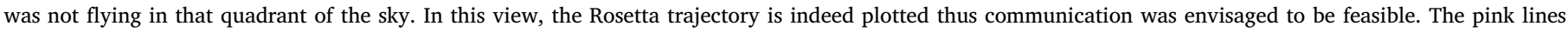

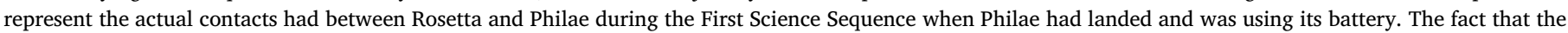

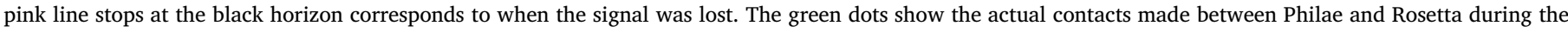

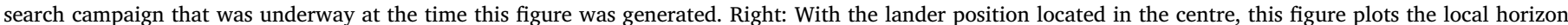

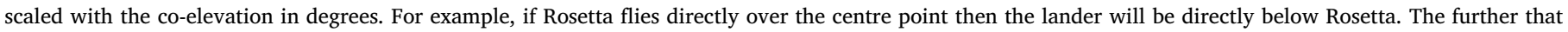

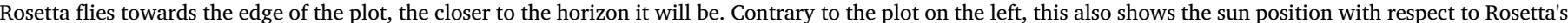

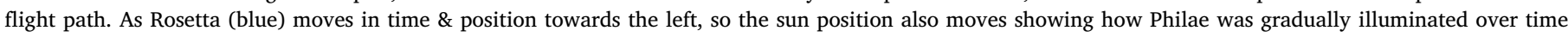

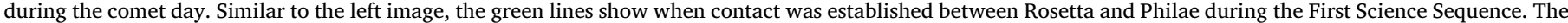

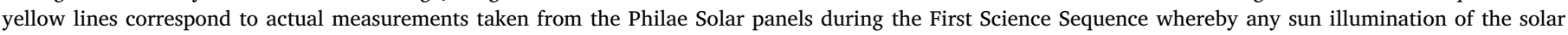

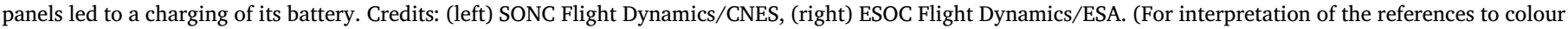
in this figure legend, the reader is referred to the Web version of this article.)

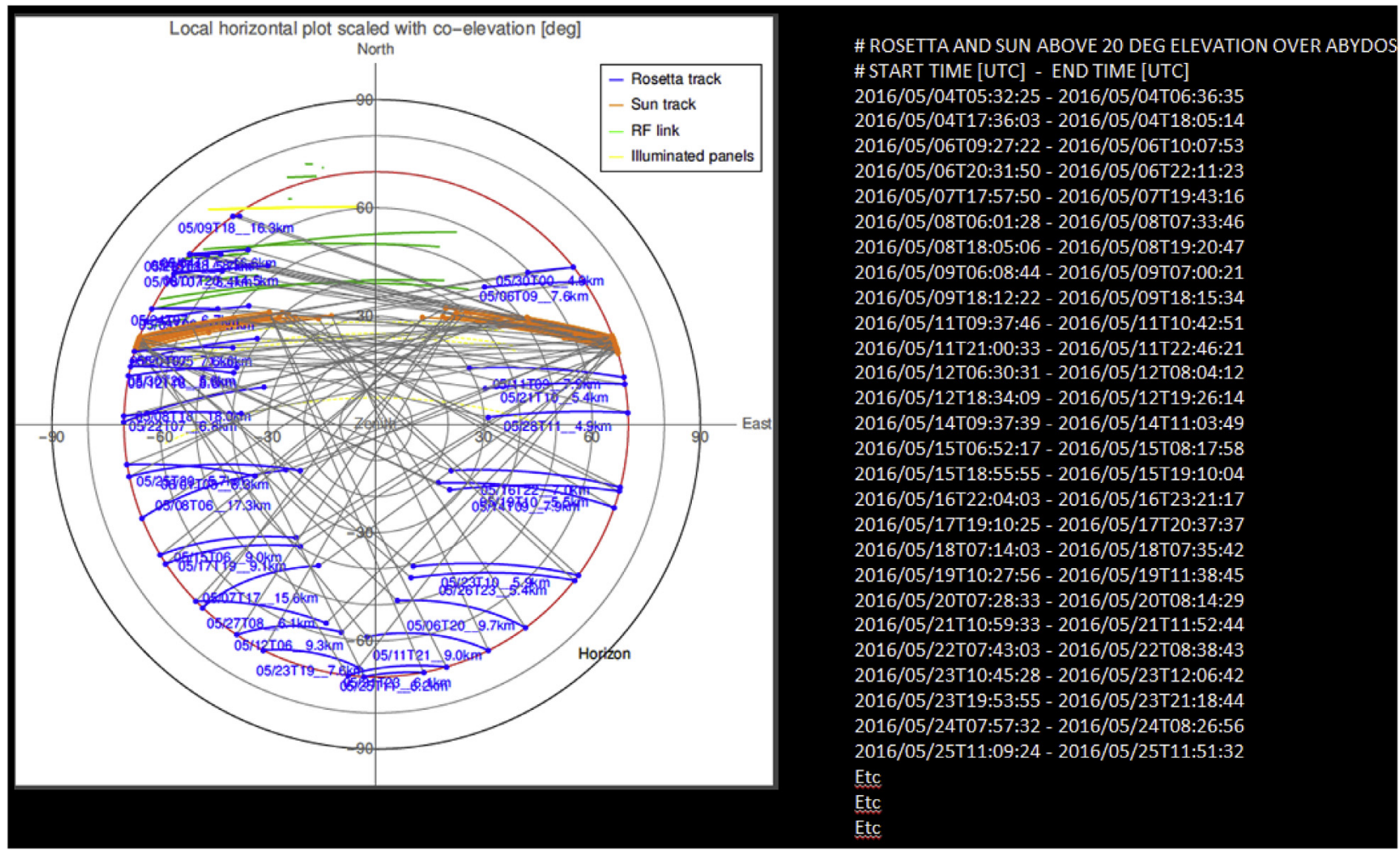

Fig. 11. Search Campaign 4 -Visualisation of the windows plotted and in ascii text. Credit: ESA/Rosetta/MOC/Flight Dynamics. 
Where the choice of a time window impacted an existing science observation then discussions would be held with the science team (SWT) to agree that this slot could be allocated for lander search.

For the case of the spacecraft maintenance windows, these related to times where spacecraft navigation was scheduled using the Navigation Cameras. Using these windows minimised impact on the scheduled instrument science activities although it became clear as time progressed that these slots were always at the end of a time slot where comet topography would block the OSIRIS camera view. In that respect, impact on science observations proved to be inevitable.

As the lander campaign progressed and more experience existed in understanding of the local topography blocking the Rosetta OSIRIS camera field of view, the selection of windows became more refined. Similarly, as Rosetta's trajectory was fixed in preparation for the end of mission descent, this placed further constraints on the remaining windows feasible during August and early September.

Once a time window was selected, there were three steps to be taken:

1. Ensure the pointing of the spacecraft was updated so that it would point exactly at the location of the lander candidate

2. Prepare the commands to be sent to the OSIRIS cameras for the time period in questions

3. Send these commands either directly to the MOC flight control team, or indirectly through the nominal mission planning process files. Once processed at the RMOC, they were uploaded to the spacecraft for execution on board.

When the data was downlinked, analysis of the images was carried out by the lander search team which included members from the OSIRIS team, from the SGS and from the SONC team.

\subsection{When is close not close enough?}

Fig. 12 shows images taken that targeted the region around the "red candidate" illustrating the challenges presented by the local scenery. There are two major features that surround the "red candidate"; a cliff overhang, and a large rocky structure that has a slightly triangular shape that was nicknamed the 'nose rock'. Add to this the fact that the edge of the Hatmahit depression was also close by and the challenge being faced becomes clear. This lander candidate was effectively "between a rock and a hard place".

At a distance of $18 \mathrm{~km}$, the images yield a scale of $\sim 30 \mathrm{~cm} /$ pixel so the lander would only be around three pixels across. To identify the lander at this distance proved to be very difficult. Even a few kilometres closer at $15 \mathrm{~km}$ the lander only appears as a bright point: the resolution was far from sufficient to make a clear identification. By mid-May 2016 the lander search team decided to only take images of the lander candidates when Rosetta was flying below a distance of $10 \mathrm{~km}$.

\subsection{Discarding imposter landers and building confidence in the prime candidate}

As Rosetta got closer to the comet, the image resolution improved, providing better views of the "red and blue candidates" but also resulted in the finding of new lander candidates in the region of interest! A high-resolution image taken on the 21st of July 2016 finally allowed to discard the "blue candidate" from the search campaign - the feature turned out to be ice on the edge of a large boulder (See Fig. 13).

It became essential to study candidates from different viewing perspectives to discard those that were clearly not the Philae lander see Fig. 14 for examples of some of the more convincing imposters.

On the 25th of May 2016, images were obtained looking over the edge of the nose rock into the cliff cavity and the prime candidate was observed to have a lander-like structure including what looked like a leg and foot (Fig. 15a). Further images taken on the 1st of June (Fig. 15b) and on the 6th of August (Fig. 15c) from a different perspective and altitude confirmed this. Indeed the orientation of this "assumed" leg and foot did compare favourably with the estimated attitude of Philae.

However, these images showed also bright reflective surfaces that could be compared with those of the lander and, unfortunately, due to icy 'lines' weaving through the cliff overhang, could also be considered to be lander-shaped ice patches. See Fig. 16.

In that respect, although the case was strong that this was really Philae, the visual resolution was insufficient to make a clear unequivocal statement to that regard. It became essential to get closer to obtain a higher resolution image as well as taking steps to gather evidence from parallel investigative studies.

\subsection{Constructing the actual horizon mask for the Philae lander}

Amongst the numerous techniques used to locate Philae on the surface, one of them proved to be quite illuminating as to its accuracy, in particular in the August 2016 timeframe when sufficient image data had been collected. This was the reconstruction of the horizon mask (line of sight from Philae across its field of view) whereby all images of the Red candidate location (whether it had been visually observed or not) were plotted on a latitude map.

This involved constructing a map of the locations around the red candidate where it was visible and not visible in the imagery, to compare with the real RF and Sun illumination data from lander contacts (Fig. 17). Where it was visible a green point was placed, where it was not visible a red point was placed. What became clear from this technique is that the "red candidate" position of the lander matched to a very high degree the RF and sun illumination data. The greyish horizon mask overlaying the edges of the outer circle in Fig. 17 needed to be adapted to match with the black line drawn on the same figure.

With confidence in the RF and sun illumination data, one could place other "lander imposters" into the line of sight map and confirm

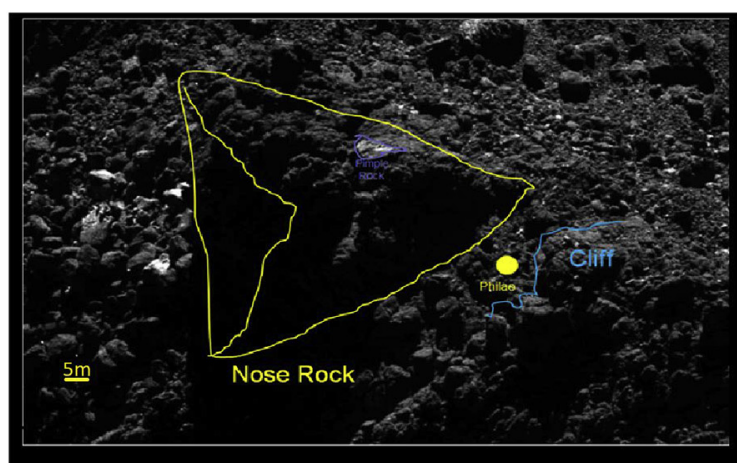

(a)

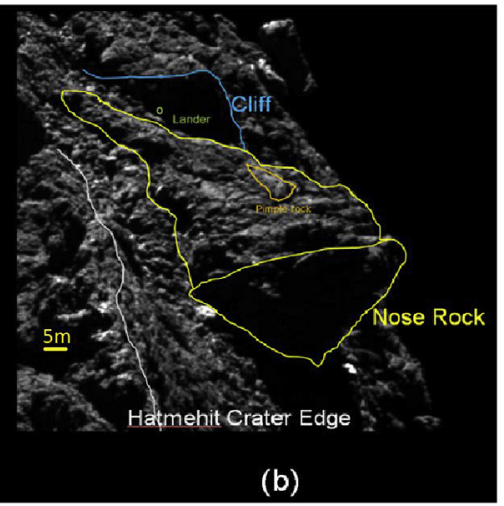

Fig. 12. Search Campaign 4 -The scenery around Philae includes a 'cliff overhang' and a large rocky structure nicknamed the 'nose rock' (labelled). An enhanced view of the region is shown, with a circle highlighting the lander candidate that becomes visible in the darkness from the reflected light emanating from the outside of the cliff. (a) is the view from above and (b) from the side. Credit: ESA/Rosetta/MPS for OSIRIS Team MPS/UPD/LAM/IAA/SSO/INTA/ UPM/DASP/IDA. Processed by ESA/ Rosetta/SGS. 


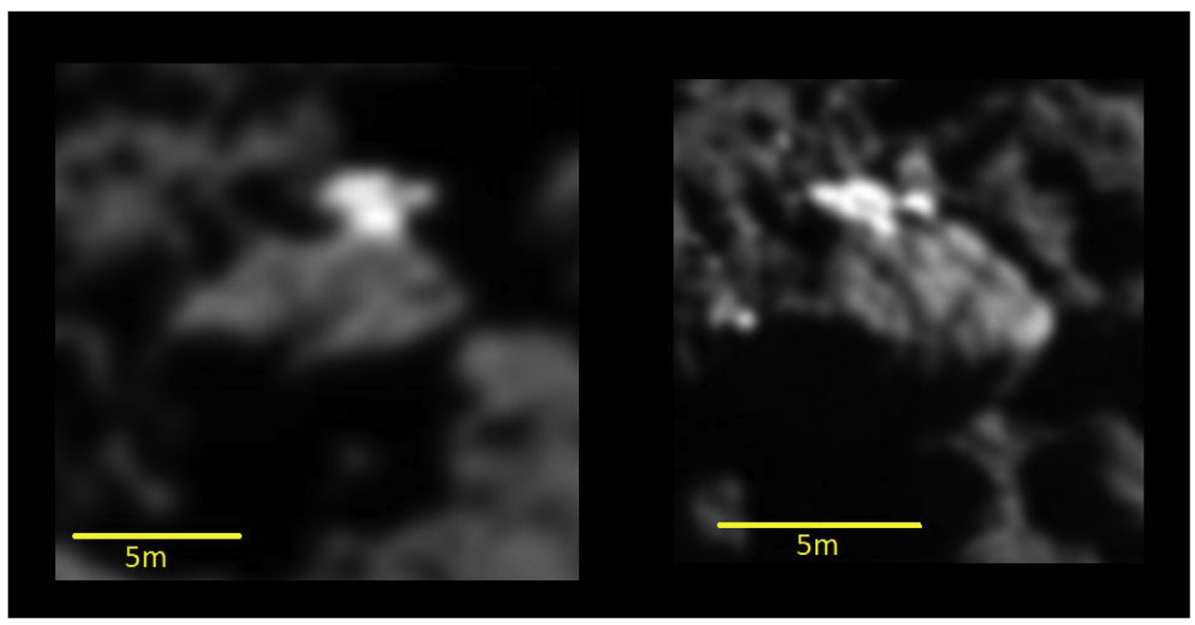

Fig. 13. Search Campaign 4 -The image on the left shows the alternate (blue) candidate as presented in Fig. 9 earlier. This candidate is discarded thanks to an image from 21st of July 2016 (right panel) that demonstrates that the bright feature observed in 2015 corresponds to a high albedo area (most likely water ice) on an outcrop. This image was taken at a distance of $8 \mathrm{~km}[15 \mathrm{~cm} /$ pixel] Credit: ESA/Rosetta/ MPS for OSIRIS Team MPS/UPD/LAM/IAA/SSO/ INTA/UPM/DASP/IDA. Processed by ESA/Rosetta/ SGS. (For interpretation of the references to colour in this figure legend, the reader is referred to the Web version of this article.)

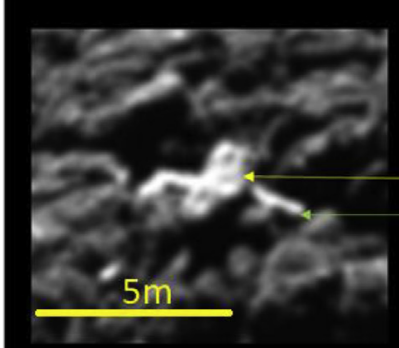

(a)

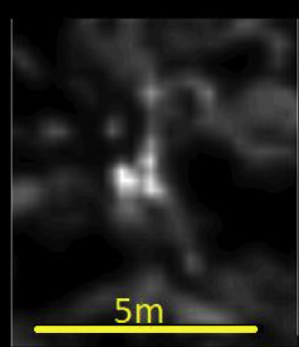

(c)

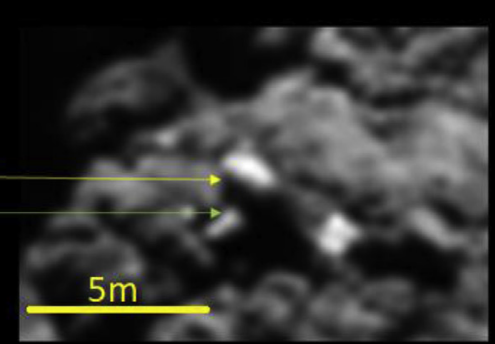

(b)

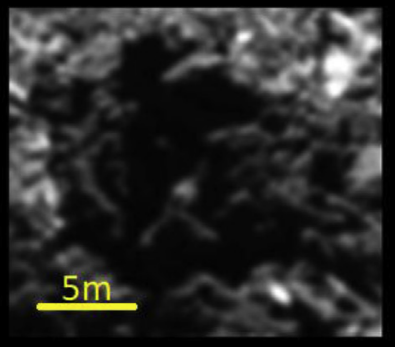

(d)

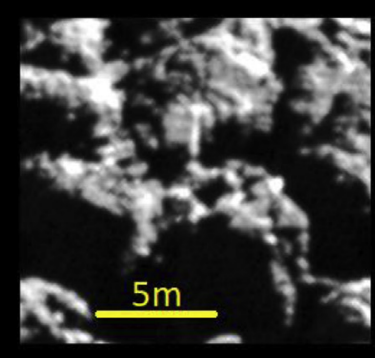

(e)

Fig. 14. Search Campaign 4 - Imposter landers. (a) and (b) show the front and overhead view of one of the lander imposters: a change in orientation from (a) to (b) reveals its true nature as an icy boulder. Images (c), (d) and (f) show ice on the front of a boulder giving a lander-like impression at first glance. Image (e) is an area of high albedo in the shape of the lander body. Credit: ESA/Rosetta/MPS for OSIRIS Team MPS/UPD/LAM/IAA/SSO/INTA/UPM/DASP/IDA. Processed by ESA/ Rosetta/SGS.

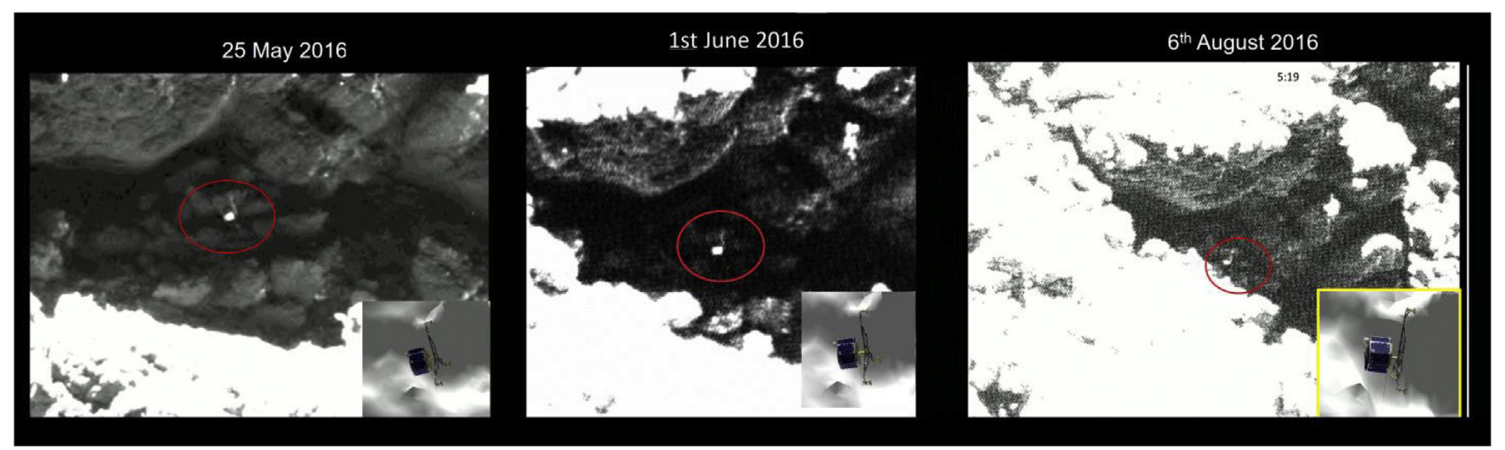

Fig. 15. Search Campaign 4 - Images taken on (a) 25th of May 2016 (b) 1st of June 2016 and (c) 6 August shown with key landscape features labelled and the lander candidate circled. A 3D model of the Philae lander with an equivalent orientation as envisaged from that viewing angle is also provided. Credit: ESA/Rosetta/MPS for OSIRIS Team MPS/UPD/LAM/IAA/SSO/INTA/UPM/DASP/IDA. Processed by ESA/Rosetta/SGS. 


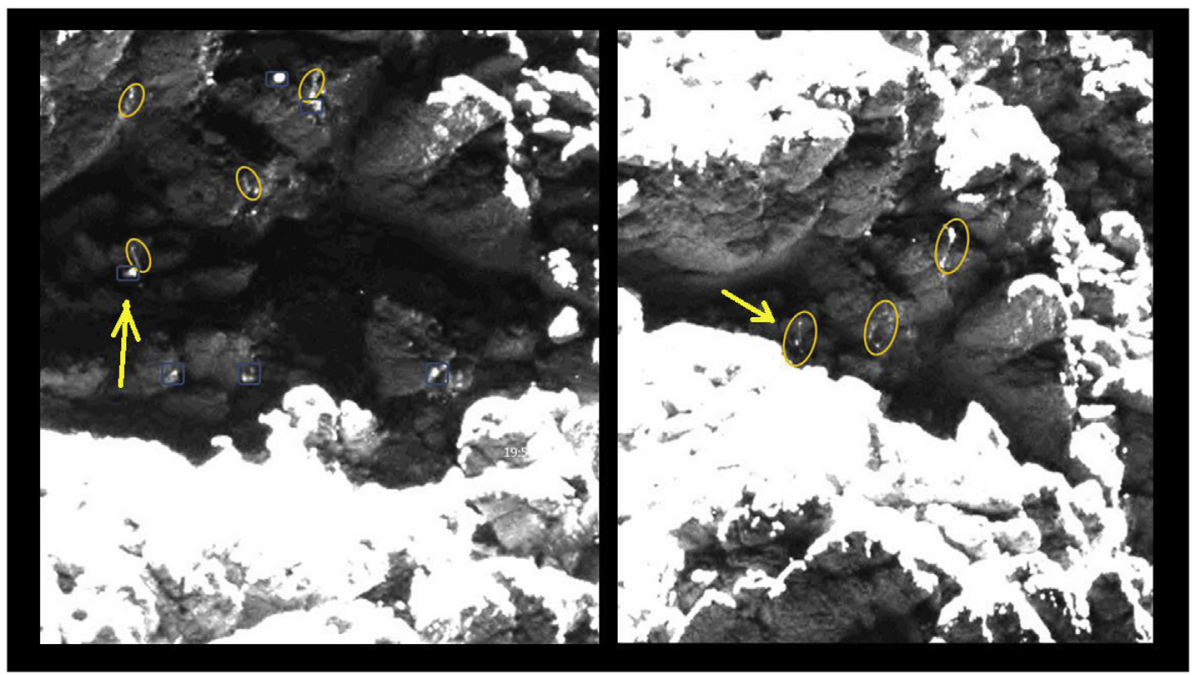

Fig. 16. Search Campaign 4 - Images taken on the 25th of May 2016 indicating that numerous linear albedo structures a few $\mathrm{m}$ in length were observed (orange ellipses) with only one of them corresponding to Philae (arrow). For scale, the length of each of Philae's legs is $1.5 \mathrm{~m}$. Credit: ESA/Rosetta/ MPS for OSIRIS Team MPS/UPD/LAM/IAA/SSO/ INTA/UPM/DASP/IDA. Processed by ESA/Rosetta/ SGS. (For interpretation of the references to colour in this figure legend, the reader is referred to the Web version of this article.)

that they did not match (Fig. 18).

\subsection{Other techniques used in support of the search}

While the focus of the campaign based itself around the use of direct image visual analysis, other key techniques and resources were used to support the search. These included:
- Checking OSIRIS images against high-resolution 3D shape models (Fig. 19)

- Using the wide dynamic range of the OSIRIS camera and taking long exposure images attempting to capture as much light as possible into the image without compressing it

Checking the best pointing and visibility opportunities against 3D shape models (Fig. 19)

Updating the local Digital Terrain Model of the CONSERT ellipse

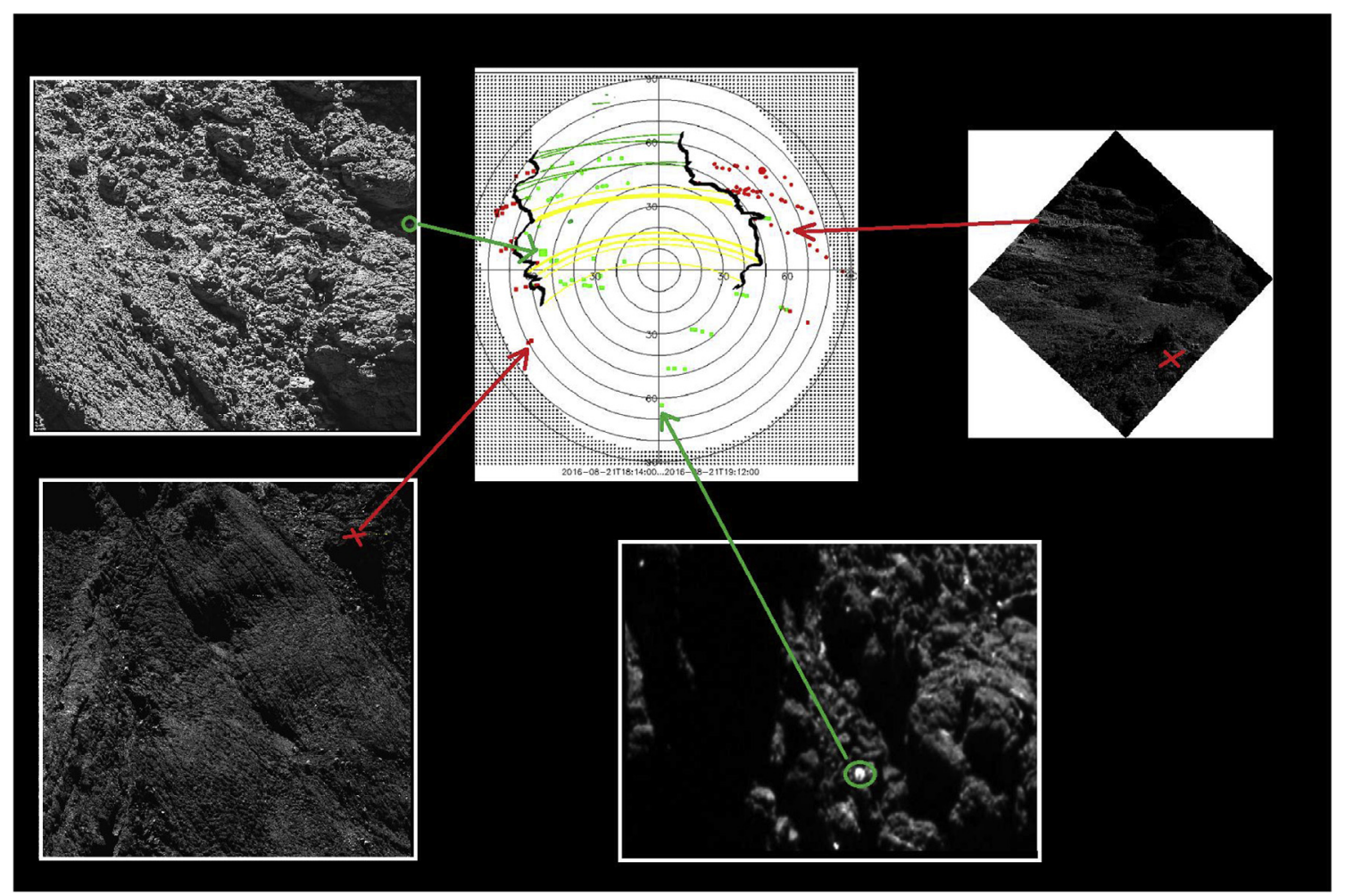

Fig. 17. Search Campaign 4 - Rosetta-Philae line-of-sight map. This image is equivalent to that presented in Fig. 10b with the "assumed" horizon mask visible on the edges in grey. Instead of the Rosetta trajectory being over-plotted, the image provides a map of green and red spots matching the visibility of the lander in the OSIRIS images taken at those positions. Green corresponds to when the lander is visible in the image e.g the top left image while red shows where an image was taken but Philae was hidden by rocks e.g. bottom left image. By joining the green spots at the edge of the image, one can get a clear view of the actual horizon mask. In this respect, at $0^{\circ}$ in the image, there was significant blocking of the lander position below $60^{\circ}$, although in the original plot this seemed to suggest full visibility. This plot also demonstrates how well the final Philae candidate position at the centre fits with the actual contacts established between Rosetta and Philae after the landing (Green lines) and the sun illumination (yellow lines). Images were taken - top right going clockwise - 14th of May 2016, 24th of July 2016, 17th of May 2016, 2nd of September 2016. Credits: ESA/Rosetta/SGS \& ESA/Rosetta/MOC/Flight Dynamics, ESA/Rosetta/MPS for OSIRIS Team MPS/UPD/LAM/IAA/SSO/INTA/UPM/ DASP/IDA. (For interpretation of the references to colour in this figure legend, the reader is referred to the Web version of this article.) 


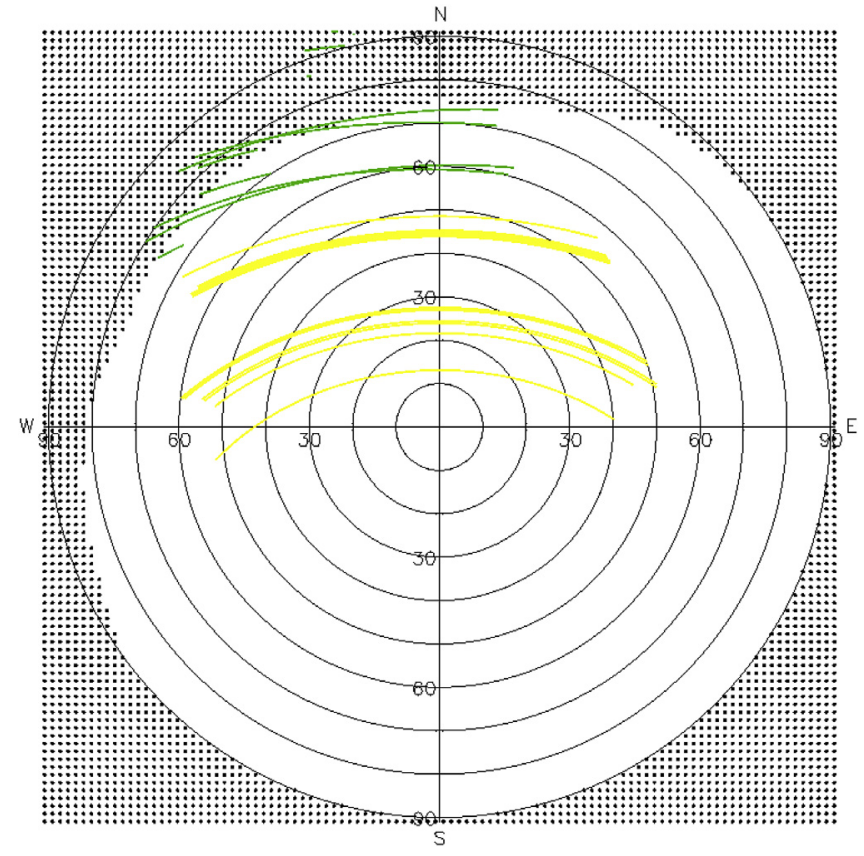

Fig. 18. Search Campaign 4 - The blue candidate with the same RF/Power plotted lines as from Fig. 13 and 17, showing there is no way that communication was feasible as some of the plotted lines are below the horizon (shaded region)- this would only 'work' if the lander had moved location. Credits: ESA/ Rosetta/SGS. (For interpretation of the references to colour in this figure legend, the reader is referred to the Web version of this article.)

region to allow better comparisons to be made, including reconstructing the view of the CIVA images [17].

- Comparing the CIVA images [18] against the high resolution OSIRIS images taken at the different lander candidate locations to look for correlations

- Kicking off a study to perform computer based automatic analysis of images to identify lander candidate.

With this combined evidence also pointing more and more towards the red candidate being the true Philae lander, it was decided to schedule more OSIRIS images to be taken in late August to attempt to see the apparent structure of the Lander "legs" previously seen in May-August images 'move' against the background terrain, by viewing it from different angles. This would confirm the structure there was indeed a manmade object rather than ice.

\subsection{Time running out - A light shining in the darkness}

As Rosetta gradually began getting closer to the comet in August and early September, and with the mission end just weeks away, the number of opportunities to get images of the red candidate were diminishing fast - and not just because of time constraints.

By late August, the red candidate was now in darkness thus the case to continue taking images of it had reduced significantly. In fact, no further image opportunities were planned for the end of August into September. With no high-resolution imagery of Philae existing at that point, the search campaign was to wrap-up by presenting all evidence gathered about the red candidate.

However, a surprising discovery was made changing that decision. In August through to September, the trajectory was such that Rosetta was gradually moving north with respect to the lander position. With this gradual move, the line of sight from Rosetta to the red candidate also moved along the edge of the nose rock (Fig. 20).

Through visual comparison with the shape model, it became clear that the 3D shape model was not a perfect tool for scheduling these images because it assumed the edge of the nose rock to be smooth and straight, when in reality it was rugged and liable to dips and peaks. There were clear mismatches between the 3D shape model and the observed local topography and, in fact, the lower part of the nose rock dipped much lower than the 3D shape model had modelled it to dip (Fig. 21).

Although the comparison using the shape model suggested that all images that could be taken on the $30^{\text {th }}$ of August and early September 2016 would have Philae hidden behind the edge of the nose rock, the visual discrepancy above was identified as being a contradiction to this belief. There remained in fact a very high chance that the Philae Lander "red candidate" was visible over the edge of the nose rock taking advantage of its ruggedness. In addition, with such image opportunities taking place when Rosetta was near its closest distance ever to the comet up to that point in time, the image resolution was expected to be exceptional.

A final set of images were scheduled to be taken on the $30^{\text {th }}$ of August, the 2nd and 5th of September on the basis that as the field of view moved along the lower edge of the nose rock, there would be a point where OSIRIS would see over it.

The problem remained that the red candidate in these last few attempts was now permanently in shadow. To offset this, the images were

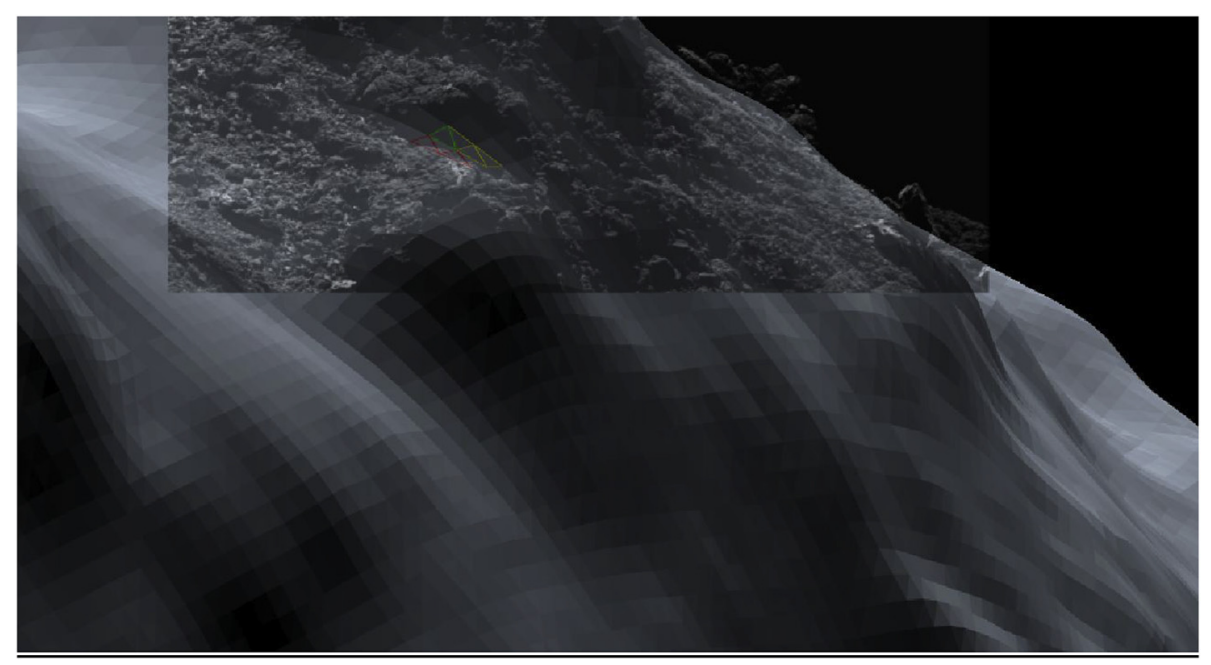

Fig. 19. Search Campaign 4 - Checking OSIRIS images (foreground inset) against 3D shape models (background). The triangular facets helped identify the location visibility. This approach is used in planning the observations as well as in checking the results afterwards. Credits: ESA/Rosetta/SGS. 


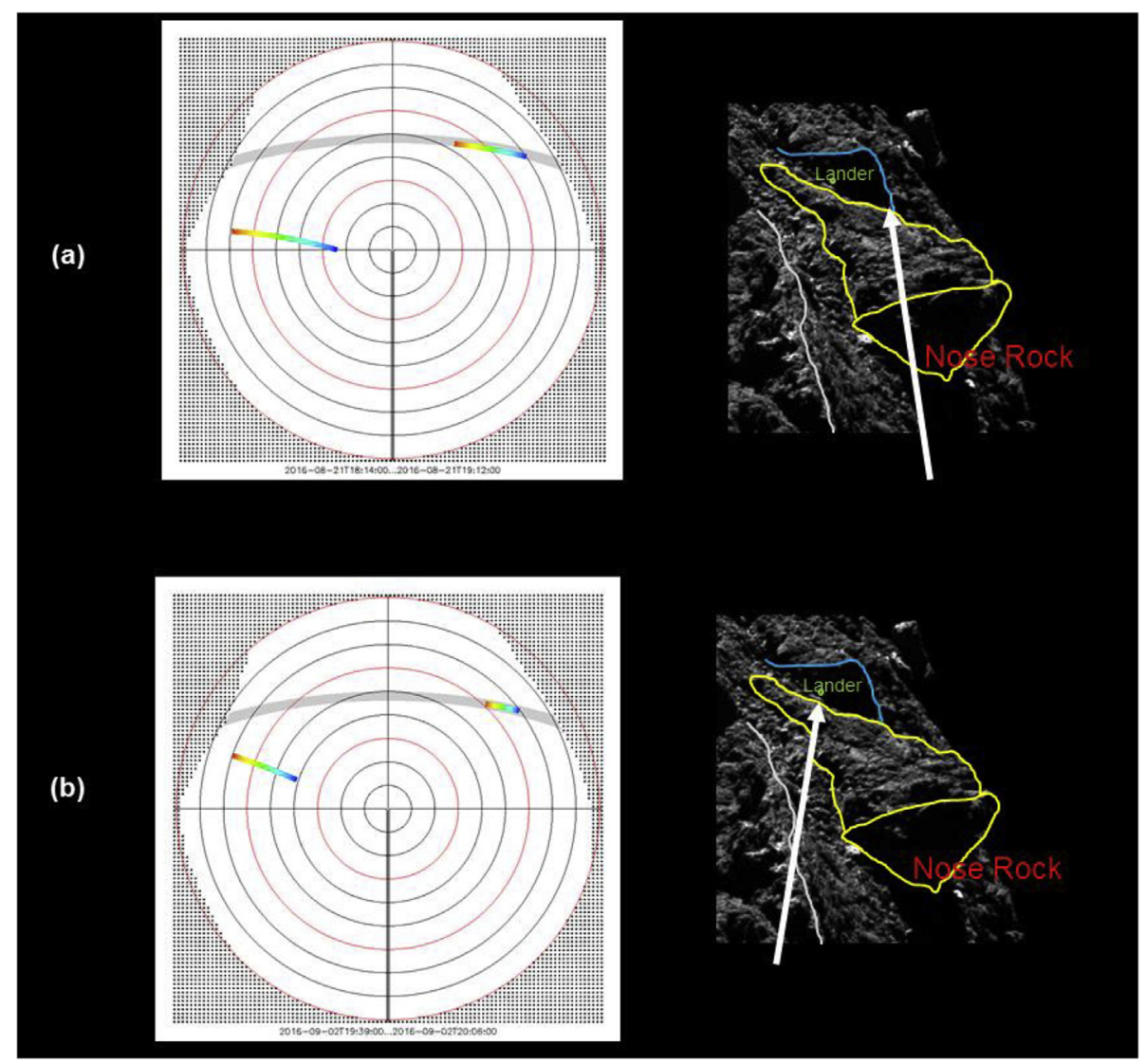

Fig. 20. Search Campaign 4 - Taking advantage of the physical outline of the nose rock. (a) Flyover plot of 21 st of August 2016 and viewing angle shown over edge of rock (b) plot of 2nd of September2016 and viewing angle over front dipped edge of rock. Credits: ESA/Rosetta/SGS based upon OSIRIS images.

scheduled to be taken as late as possible in the slot to improve the reflected light coming from the outside of the cliff. In addition, the OSIRIS team members decision to use the wide dynamic range of the camera to take long exposure images attempting to capture as much light as possible into the image without compressing it, proved crucial in seeing into, what was now, darkness.

On the $30^{\text {th }}$ of August 2016, the leg of the lander could be observed just over the edge of the rock. Note that this image came down a number of days after the 2nd of September image due to how the downlinking of OSIRIS on-board storage data was prioritised on-board Rosetta.

On the 2nd of September 2016, the orbiter line of sight was adequate to view over the front edge of the rock and high-resolution images were taken. The distance to the comet surface was $2.7 \mathrm{~km}$ and the resolution approximately $5 \mathrm{~cm}$ /pixel. Upon enhancing the images, a light shone through the darkness - the Philae lander was seen in high resolution on its side on the surface of the comet (Fig. 22 and Fig. 23).

But there remained one final factor at play that nearly made this

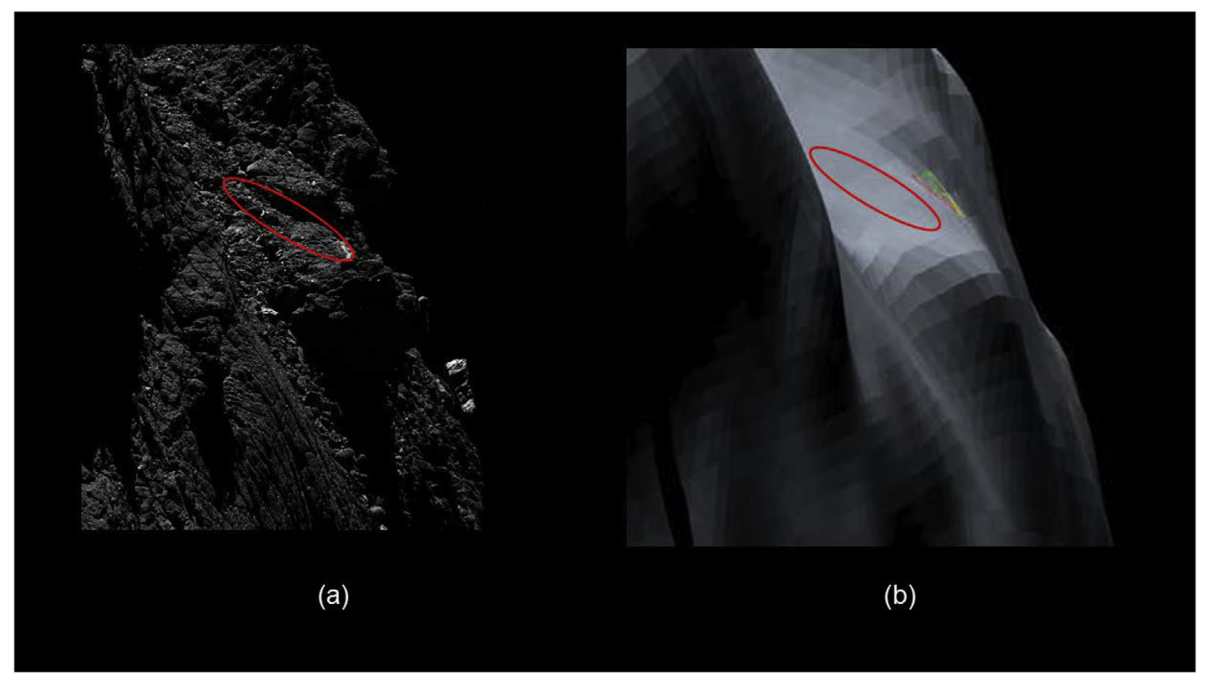

Fig. 21. Search Campaign 4 - Real edge of nose rock versus the simulated edge of the 3D model - image from the 21st of August 2016. Credits: ESA/Rosetta/SGS based upon OSIRIS images. 


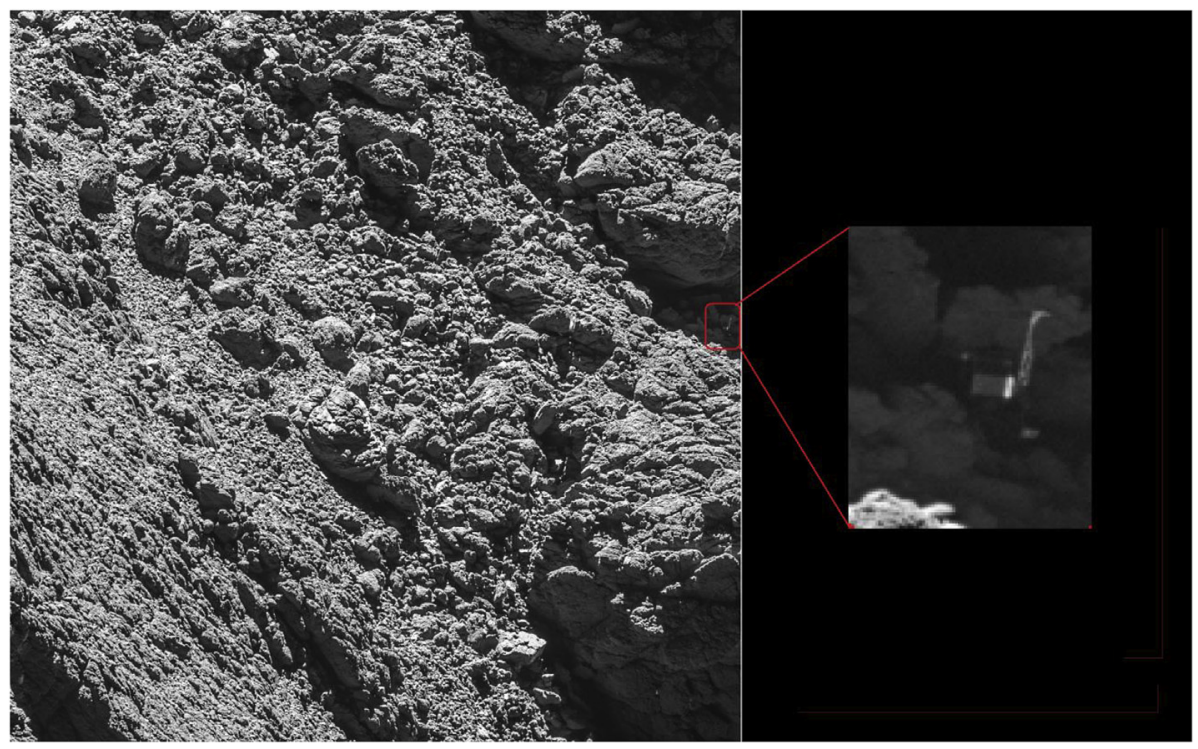

Fig. 22. Search Campaign 4 - Philae on the comet taken on the 2nd of September 2016 from a distance of $2.7 \mathrm{~km}$. The image scale is about $5 \mathrm{~cm} /$ pixel. The image shows Philae's $1 \mathrm{~m}$-wide body and two of its three legs can be seen extended from the body. The images also provide proof of Philae's orientation. Credit: ESA/Rosetta/MPS for OSIRIS Team MPS/ UPD/LAM/IAA/SSO/INTA/UPM/DASP/IDA. Processed by ESA/Rosetta/SGS.

now-famous image another miss. In Fig. 22, it can be seen that instead of the lander being nicely framed in the centre of the image, which was the plan, it is skewed all the way over on the right hand side. This "offpointing" was due to the close distances of the Rosetta flyovers whereby the trajectory of the spacecraft was affected by the higher gravity perturbation and drag from cometary gas at very low altitudes along with normal manoeuvre uncertainties.

To overcome this and in order to point the instruments to the Philae location the RMOC Flight Dynamics team needed to predict very accurately the spacecraft position with respect to the comet for the whole commanded period. In the case of the 2nd of September Philae image, this trajectory prediction was performed more than $36 \mathrm{~h}$ before the image was taken achieving an exceptional accuracy of $\sim 50 \mathrm{~m}$ at the time of the image. Thus although Philae ended up not being in the centre of the image, the fact that Philae was in the camera field of view at all and not many degrees away, reflected the excellent performance of the ESOC Flight Dynamics team.

\subsection{A successful "FINAL" image from the lander search campaign}

When the images from the 2nd of September 2016 were downlinked to Earth on the 4th of September, an initial analysis by the OSIRIS team confirmed that a high resolution image of the Philae lander had finally been obtained. Located in the corner of the image, it was confirmed to be the red candidate that had been imaged intensively during the previous months.

The lower resolution images taken previously of the red candidate, which had hinted at a lander-like structure, were confirmed retrospectively to be of the Philae lander. The red candidate, also known as the LAM candidate, was finally confirmed to be the Philae. Moreover, Philae was indeed inside the CONSERT radar triangulation result (see Fig. 24) and its attitude was close to that derived by the ROMAP team [23] and the Philae Lander team [13,19,24]. The image also proved that the lander could not have moved significantly since it landed at Abydos in November 2014.

As regards the image opportunity which took place on the 5th of September, off-pointing led to the lander falling outside the field of view of the image. In that respect, that image of the 2nd of September, showing the lander on the surface of the comet was in fact the LAST successful image taken by the lander search group of that spot before Rosetta concluded its mission on $30^{\text {th }}$ of September 2016.

The identification took place when the comet, Philae and Rosetta, were at a distance of approximately 680 million kilometres from the Earth, some 22 months after Philae's touchdown.

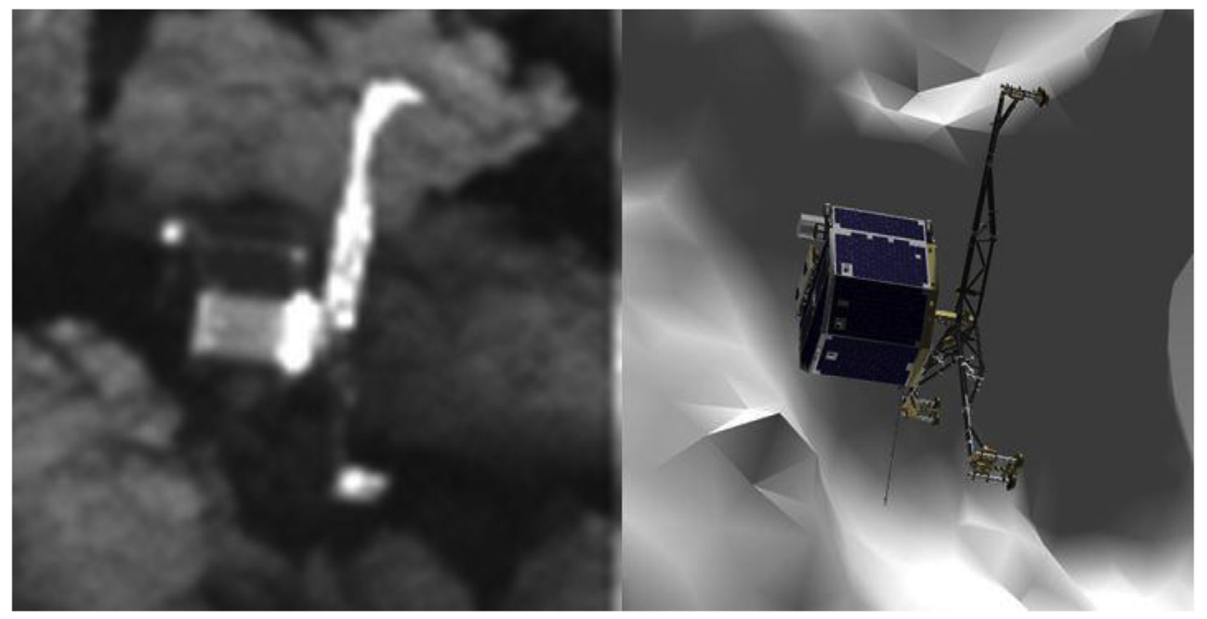

Fig. 23. Search Campaign 4 - Zoomed in image of Fig. 22 with 3D model comparison of Philae beside it. Credit: Left hand image - ESA/Rosetta/MPS for OSIRIS Team MPS/UPD/LAM/IAA/SSO/INTA/UPM/DASP/IDA. Right hand image: CNES/SONC. 


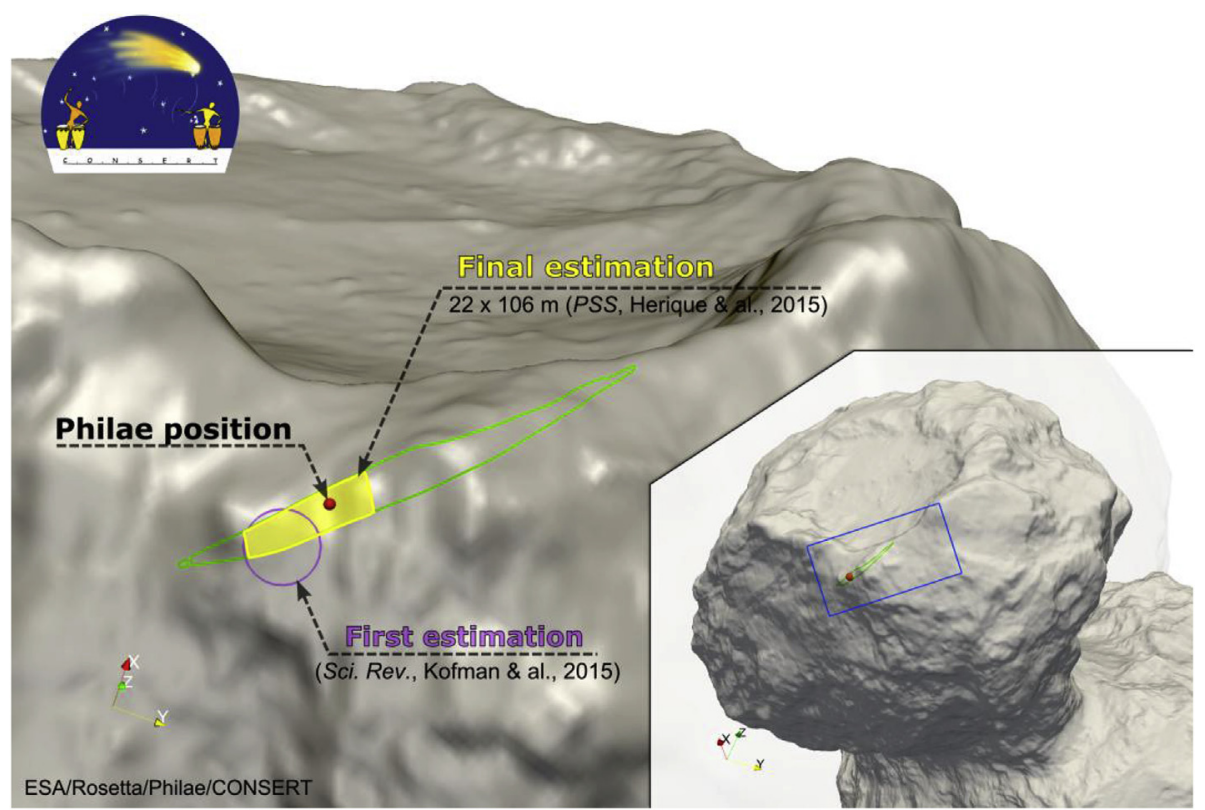

Fig. 24. Search Campaign 4 - Lander localisation inside CONSERT search ellipse. Credits: Superimposed ellipse: ESA/Rosetta/Philae/CONSERT; Shape model: ESA/ Rosetta/MPS for OSIRIS Team MPS/UPD/LAM/IAA/SSO/INTA/UPM/DASP/IDA.

\section{Conclusions}

During the course of the exercise, a number of lessons were identified which are recorded here.

- The positioning of a number of LEDs on the Philae lander would have made the search for the lander much easier.

- The CONSERT team were able to programme their instrument to perform a non-science measurement that served to help in the search of the final location. The capability to be able to adapt the existing software to support urgent modes is recommended.

- The use of a 3D shape model is highly recommended but it has its limitations in the number of facets in use when trying to find an object located in one of those facets.

- The search campaign was based on the 2D drawing on shape model, with calculations done in latitude and longitude. Errors linked to the shape model in use could change drastically the positions and the Cartesian to lat/long conversions, especially in the case of high-incidence images. Data processing and operations planning have to take care of this point.

With the lander finally located the lander search group wound up its activities in September 2016. There is no doubt that without the significant work, drive and efforts of all teams and individuals involved in the lander search group - mixed with some well-deserved good luck the lander would not have been imaged before Rosetta made its own touchdown on the surface of the comet, on the $30^{\text {th }}$ of September 2016.

\section{Acknowledgements}

In addition to those mentioned specifically in the co-author list who belong to the centres below, the following individuals are recognised for their work during the course of the 22 months of the search for the Philae Lander:

CONSERT Team: P.Pasquerro, P.Puget, S.Zine.

Lander Control Centre Team: C.Fantinati, J.Biele, S.Jansen, O.Kuechemann, B.Cozzoni, F.Finke, V.Lommatsch, M.Maibaum.

Lander Steering Group: U.Christensen, J.B.Dubouis, E.Flamini, G. Haerendel, F.jansen, P.Kletzkine, H.E.Koskinen, F.Rocard, H.Scheuerle, S.Szalai, B.Feuerbacher, R.Young, F.Huber, G.Schwehm
OSIRIS team: The entire OSIRIS team is represented by the PI and the lead scientists (H. Sierks, G. Naletto, P. L. Lamy, R. Rodrigo, D. Koschny, B. Davidsson) and the team of Co-Is, associate scientists and assistants.

RMOC Team: A.Hubault, T.Francisco, M.Eiblmaier, R.Kay, A.Rijiranuwat, M. Mueller,Y. Enginger,A. Rivero, J. M. Garcia, F. Zuiani, C. Aldebert, A. Martinez, ROMAP-MAG team: P.Heinisch, HU.Auster, I.Richter, D.Hercik, K.H.Glassmeier

RSGS team: C.Vallat, M.Kueppers, M.P.Ayucar, R.Hoofs.

SONC team: AMoussi-Soffys, C.Delmas, J.Durand, E.Jurado, E.Canalias, A.Blazquez, T.Martin, T.Bloch, C.Agard, D.Hallouard, For the case of CONSERT, Support from the Centre National d'Etudes Spatiales (CNES, France) for this work, based on observations with CONSERT on board Rosetta, is acknowledged. The CONSERT instrument was designed built and operated by IPAG, LATMOS and MPS and was financially supported by CNES, CNRS, UGA, DLR and MPS.

Rosetta is an ESA mission with contributions from its Member States and NASA. Rosetta's Philae lander is provided by a consortium led by DLR, MPS, CNES and ASI. The authors thank the teams of Rosetta (SGS and ESOC) and Philae (LCC and SONC) for making the CONSERT operations possible.

\section{Appendix A. Supplementary data}

Supplementary data to this article can be found online at https:// doi.org/10.1016/j.actaastro.2018.12.035.

\section{References}

[1] K.H. Glaßmeier, H. Böhnhardt, D. Koschny, E. Kührt, I. Richter, The Rosetta mission. Flying towards the origins of the solar system, Space Sci. Rev. 128 (2007) $1-21$.

[2] S. Ulamec, J. Biele, A. Blazquez, B. Cozzoni, C. Fantinati, P. Gaudon, K. Geurts, E. Jurado, O. Küchemann, V. Lommatsch, M. Maibaum, H. Sierks, L. Witte, Rosetta lander - Philae: landing preparations, Acta Astronom. 107 (2015) 79-86.

[3] S. McKenna-Lawlor, P. Rusznyak, J. Balaz, W. Schmidt, C. Fantinati, O. Kuechemann, K. Geurts, Performance of the mission critical Electrical Support System (ESS) which handled communications and data transfer between the Rosetta Orbiter and its Lander Philae while en route to and at comet 67P/, ChuryumovGerasimenko Acta Astron. 125 (2016) 118-136.

[4] J. Biele, et al., The landing(s) of Philae and inferences about comet surface mechanical properties, Science 349 (2015) aaa9816.

[5] Special Issue in Science 349, 2015. 
[6] J.P. Bibring, Y. Langevin, J. Carter, P. Eng, B. Gondet, et al., 67P/ Churyumov-Gerasimenko surface properties as derived from CIVA panoramic images, Science 349 (2015) aab 0671.supplementary material.

[7] C. Dudal, C. Loisel, Rosetta-Philae RF link, challenging communications from comet, Acta Astronom. 125 (2016) 137-148.

[8] H.U. Keller, et al., OSIRIS - the scientific camera system onboard Rosetta, Space Sci. Rev. 128 (2007) 433-506.

[9] The Rosetta Navigation Camera description, https://imagearchives.esac.esa.int/ index.php?/page/rosetta navcam.

[10] J.P. Bibring, et al., CIVA : comet infrared and visible analyser, Space Sci. Rev. 128 (2007) 397-412.

[11] H.U. Auster, et al., ROMAP : Rosetta magnetometer and plasma monitor, Space Sci. Rev. 128 (2007) 221-240.

[12] W. Kofman, et al., The comet nucleus sounding experiment by Radiowave transmission (CONSERT), Space Sci. Rev. 128 (2007) 413-432.

[13] S. Ulamec, C. Fantinati, M. Maibaum, K. Guerts, J. Biele, S. Jansen, O. Kúchemann, B. Cozzoni, F. Finke, V. Lommatsch, A. Moussi-Soffys, C. Delmas, L. O'Rourke, Rosetta lander - landing and operations on comet 67P/Churyumov-Gerasimenko, Acta Astronom. 125 (2016) 80-91.

[14] P. Muñoz, F. Budnik, V. Companys, B. Godard, C. Casas, T. Morley, V. Janarthanan, Rosetta navigation during lander delivery phase and reconstruction of Philae descent trajectory and rebound, Proceedings $25^{\text {th }}$ International Symposium on Space Flight Dynamics - 25th ISSFD, Munich, Germany, 2015.

[15] P. Lamy, G. Faury, L. Jorda, et al., Identification and Characterization of the Landing Site of Philae from OSIRIS-NAC Images, vol. 10, (2015) EPSC 2015-783.

[16] R. Garmier, T. Ceolin, T. Martin, A. Blazquez, E. Canalias, E. Jurado, et al., Philae landing on comet churyumov-gerasimenko: understanding of its descent trajectory, attitude, rebound and final landing site, Proceedings 25th International Symposium on Space Flight Dynamics - 25th ISSFD, Munich, Germany, 2015.

[17] C. Capanna, L. Jorda, P. Lamy, et al., Extreme 3D reconstruction of the final ROSETTA/Philae landing site, EGU General Assembly 2016, Geophys. Res. Abstr. 18 (2016).

[18] Remetean, et al., Philae locating and science support by robotic vision techniques, Acta Astronaut. 125 (2016) 161-173.

[19] K. Geurts, C. Fantinati, S. Ulamec, R. Willnecker, Rosetta lander: on-comet operations execution and recovery after the unexpected landing, Proceedings of the 14th International Conference on Space Operations, Conference, Korea, 2016, pp. 16-20.

[20] Balszs, et al., Command and data management system (CDMS) of the Philae lander, Acta Astronom. 125 (2016).

[21] W. Kofman, A. Herique, Y. Barbin, J. Barriot, V. Ciarleti, et al., Properties of the 67P/Churyumov-Gerasimenko interior revealed by CONSERT radar, Science 349 (2015) aab0639.

[22] A. Herique, Y. Rogez, O.P. Pasquero, S. Zine, P. Puget, W. Kofman, Philae localization from CONSERT/Rosetta measurement, Planet, Space Sci 117 (2015) 475-484.

[23] Heinisch, P. Auster, H-Ul, Richter, I. Hercik, D. et al. Attitude reconstruction of ROSETTA's Lander PHILAE using two-point magnetic field observations by ROMAP and RPC-MAG, Acta Astronom., Vol 125, p. 174-182.

[24] S. Ulamec, L. O'Rourke, J. Biele, B. Grieger, R. Andres, S. Lodiot, P. Muñoz, A. Charpentier, S. Mottolo, J. Knollenberg, M. Knapmeyer, et al., Rosetta Lander Philae: Operations on comet 67P/Churyumov-Gerasimenko, analysis of wake-up activities and final state, Acta Astronaut. 137 (2017) 38-43. 